\title{
Coaxial multimode cavities for fundamental superconducting rf research in an unprecedented parameter space
}

\author{
P. Kolb $\odot,{ }^{1, *}$ Z. Yao, ${ }^{1}$ T. Junginger $\odot,{ }^{1,2}$ B. Dury $\odot,{ }^{3}$ A. Fothergill $\odot,{ }^{3}$ \\ M. Vanderbanck, ${ }^{3}$ and R. E. Laxdal ${ }^{1}$ \\ ${ }^{1}$ TRIUMF, 4004 Wesbrook Mall, Vancouver, British Columbia V6T 2A3, Canada \\ ${ }^{2}$ University of Victoria, Victoria, British Columbia, Canada \\ ${ }^{3}$ University of British Columbia, Vancouver, British Columbia, Canada
}

(Received 19 August 2020; accepted 18 November 2020; published 2 December 2020)

\begin{abstract}
Recent developments in superconducting radio-frequency (SRF) research have resulted in significant increases of cavity performance. Advances have been made in both reducing rf surface resistance and pushing the readily achievable accelerating gradient by using novel SRF cavity treatments including surface processing, custom heat treatments, and flux expulsion. These developments have been largely driven by specific tests on single-cell elliptical cavities, while TEM cavities have typically been designed and developed as specific to a project application and not as standard test articles that could be replicated across various labs. To address this, two purpose-built research cavities, one quarter-wave and one halfwave resonator, have been designed and built to allow characterization of TEM-mode cavities with standard and novel surface treatments. The cavities are intended as the TEM-mode equivalent to the $1.3 \mathrm{GHz}$ single-cell cavity, which is the essential tool for high-frequency cavity research. Given their coaxial structure, the cavities allow testing at the fundamental mode and higher harmonics, giving unique insight into the role of $\mathrm{rf}$ frequency on fundamental loss mechanisms from intrinsic and extrinsic sources. In this paper, the cavities and testing infrastructure are described, and the first performance measurements of both cavities are presented. Temperature-dependent surface resistance data are analyzed to extract both the temperature-dependent and temperature-independent components and their dependence on the rf field and frequency. In particular, the temperature-dependent component was found to be at low fields $\propto \omega^{1.8(1)}$ at $4.2 \mathrm{~K}$ and $\propto \omega^{1.7(2)}$ at $2 \mathrm{~K}$, agreeing fairly well with the theoretical model. The growth of the temperaturedependent surface resistance with increasing field amplitude matches both exponential and quadratic growth models fairly well in the examined range. The independent component is determined to be $\propto \omega^{0.7(1)}$, matching roughly with anomalous losses, while no clear field dependence was determined. In addition, first measurements of a $120^{\circ} \mathrm{C}$ baking treatment and of the external magnetic field sensitivity are presented.
\end{abstract}

DOI: 10.1103/PhysRevAccelBeams.23.122001

\section{INTRODUCTION}

Nuclear physics experiments rely on superconducting radio-frequency (SRF) heavy ion particle accelerators such as the Isoptope Separator and ACelerator (ISAC)-II [1] facility at TRIUMF to study the nuclear structure of rare isotopes among other topics of research. New large driver accelerators for hadron facilities such as Facility for Rare Isotope Beams [2], Rare isotope Accelerator complex for ON-line experiment [3], Proton Improvement Plan-II [4], European Spallation Source [5,6], and Chinese Accelerator

\footnotetext{
*kolb@triumf.ca

Published by the American Physical Society under the terms of the Creative Commons Attribution 4.0 International license. Further distribution of this work must maintain attribution to the author(s) and the published article's title, journal citation, and DOI.
}

Driven System [7] are being installed or developed to support a variety of research interests. To increase the energy of the beam in the velocity regime up to $\beta \leq 0.6$, these accelerators use different types of TEM-mode SRF cavities, such as quarter-wave resonators (QWRs) and halfwave resonators (HWRs) at frequencies ranging from 80 to $400 \mathrm{MHz}$. SRF research is essential to advance particle accelerator technology. Higher gradients result in shorter, more economical linear accelerators (linacs) or higher energies for the same accelerator length. As these SRF cavities are typically cooled with liquid helium at temperatures near 2.0 or $4.2 \mathrm{~K}$, the rf losses in the cavity walls are a major cost driver in capital investment and in operating budget for the cryoplant and its infrastructure. Higher quality factors $Q_{0}$ mean smaller cryoplants can be used for the same amount of accelerating voltage. Despite the strong interest in TEM-mode cavities for new hadron projects, the bulk of the recent developments to enhance cavity performance have been performed on high- $\beta$, 
1.3 GHz elliptical cavities in support of large projects such as European X-Ray Free-Electron Laser [8], Linear Coherent Light Source (LCLS)-II [9], and future projects such as the International Linear Collider [10,11]. In recent years, significant advances have been made both in enhancing the quality factor $Q_{0}$, which corresponds to a lowered surface resistance $R_{s}$, but also in pushing the readily achievable accelerating gradient $E_{\text {acc }}$ to higher levels by using novel SRF cavity treatments with a focus on improved surface processing, customized heat treatments, and a better understanding of flux expulsion [12-18]. These advances have come almost exclusively on standard single-cell elliptical cavities that reside in many labs around the world. These cavities are relatively inexpensive and allow straightforward comparison of global results. Adoption of these new treatments into TEM-mode cavities has been slowed somewhat by the lack of a similar test cavity. While research and development efforts have been done, the focus is more on applying known recipes to customized project cavities in order to reproducibly meet the project requirements and not driven by understanding fundamental limitations.

This paper is structured as follows: Section II motivates the development of the presented SRF cavities and tools. Section III will describe the cavity design and goes over details of the surface preparation, testing methodology, and available infrastructure. Section IV shows the cavity performance as a function of peak surface field $B_{p}$ for a conventional surface treatment. Also, $Q_{0}(T)$ data collected during the cooldown from 4 to $2 \mathrm{~K}$ are analyzed. In addition, performance measurements of the QWR after $120^{\circ} \mathrm{C}$ baking are presented, as well as flux sensitivity data are shown for the QWR as an example of characterizations of flux expulsion from TEM-mode cavities. Section V presents a summary of the presented work, including an outlook into future work.

\section{MOTIVATION}

Much of the research for $1.3 \mathrm{GHz}$ applications has been done on single-cell cavities. These are compact cavities not intended for acceleration but designed with similar features such as rf frequency, peak surface field to accelerating gradient ratios, and identical accelerating mode to the typical nine-cell variants designed for on-line acceleration. Singlecell cavities are relatively inexpensive and have been duplicated around the world to allow treatment comparison between research centers throughout the SRF community and greatly enhance development progress.

For TEM-mode cavities, such a focused global development is much more difficult, since the design space is broader in terms of hadron velocity and rf frequency. Projects typically optimize cavity parameters within the project and design a few unique cavity designs to span the intended velocity range of a particular linac.

Therefore, a TEM-mode cavity with an equivalent role to the single-cell elliptical cavity does not exist. This lack of a test cavity motivated the development of a set of coaxial test cavities analogous to the $1.3 \mathrm{GHz}$ single-cell cavity. These cavities allow one to shift SRF research on low $\beta$ cavities away from project-driven designs to a more focused study of the TEM geometry and frequency range and offer a systematic way to enhance cavity performance and study fundamental SRF characteristics. In addition, the coaxial geometries can be tested not only at the fundamental eigenmode but also at higher harmonics, enabling datasets at several rf frequencies within the same cooldown cycle and for the same cavity treatment, surface roughness, material properties, and other environmental conditions such as external magnetic fields, allowing frequencyresolved observations of the cavity performance. In combination with the available infrastructure and tools available at TRIUMF, these cavities allow exploration of a previously unavailable parameter space for characterizing SRF cavities without changing intrinsic and extrinsic factors to the cavity.

New heat treatments developed on $1.3 \mathrm{GHz}$ single-cell cavities and rolled out on production nine-cell units have not been employed on TEM-mode cavities on a large scale except for degassing at $650^{\circ}-800^{\circ} \mathrm{C}$ and the $120^{\circ} \mathrm{C}$ in situ vacuum bake [19]. Some early results of implementing new treatments like nitrogen infusion on coaxial cavities show promise [20] for performance improvements; especially at $2 \mathrm{~K}$, a significant increase in $Q_{0}$ and a reduction of $Q$ slope in the medium field range have been observed, unlike the $120^{\circ} \mathrm{C}$ bake, which seems to improve performance in lowfrequency cavities at $4 \mathrm{~K}$ but has a deteriorating effect at 2 K [21]. Systematic studies on flux expulsion have been instrumental in reaching high quality factors in elliptical cavities and are now being conducted on TEM-mode cavities $[22,23]$. These studies reveal that the geometry of the cavity, the external field distribution, and the temperature distribution during the superconducting transition are important factors in flux expulsion in order to reach high quality factors.

Several open questions remain concerning the performance of TEM-mode cavities. What is the source of the medium field $Q$ slope at $4.2 \mathrm{~K}$ that has forced some projects to choose operation at 2 over $4.2 \mathrm{~K}$, despite the reduced losses that come with low frequency and added technical complexity of $2 \mathrm{~K}$ operation? What customized heating or doping treatments optimized for $1.3 \mathrm{GHz}$ would help to lower the surface resistance $R_{s}$ at $4 \mathrm{~K}$ for low-frequency TEM-mode cavities? How does the cavity geometry influence magnetic flux trapping and expulsion? Is there a flux expulsion method that would benefit TEM-mode cavities to lower the residual resistance? Using a dedicated purpose-built set of coaxial cavities allows tackling these questions, advancing the understanding of TEM-mode cavities, and shedding light on the role of the rf frequency in cavity performance in a systematic way.

This paper reports on the design and first performance characterizations of two coaxial test cavities: a quarter-wave 


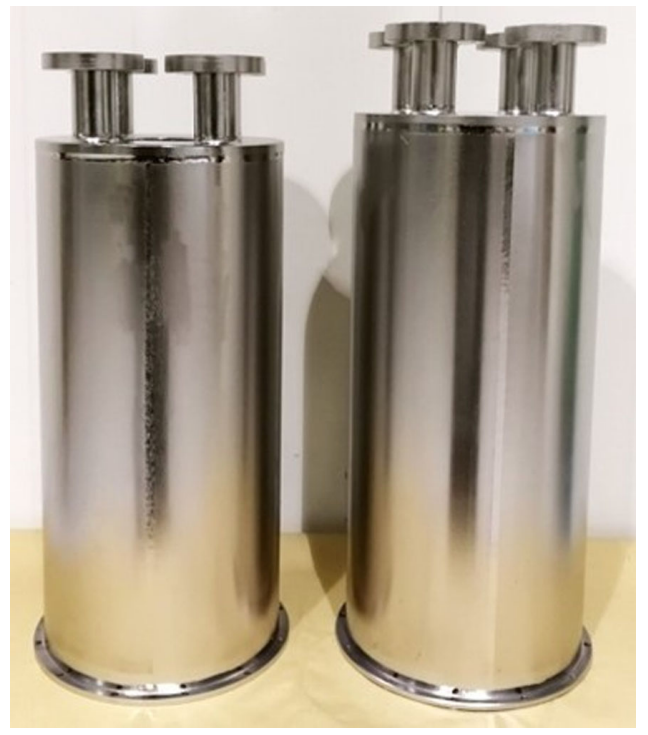

FIG. 1. Photo of the two coaxial cavities.

resonator and a half-wave resonator, shown in Fig. 1. These two cavity geometries represent the two main structure types used in hadron linacs to date. Each cavity is designed to operate in the fundamental and several higher-order modes (HOMs) with similar rf characteristics in terms of peak surface field ratio $E_{p} / B_{p}$. The field distribution of the fundamental mode and HOMs of interest for the two cavities are shown in Fig. 2. The two resonators are intended to be used for a broad array of fundamental studies. These studies include the measurement of $\mathrm{rf}$ surface resistance as a function of peak magnetic surface field $B_{p}$ and temperature $T$
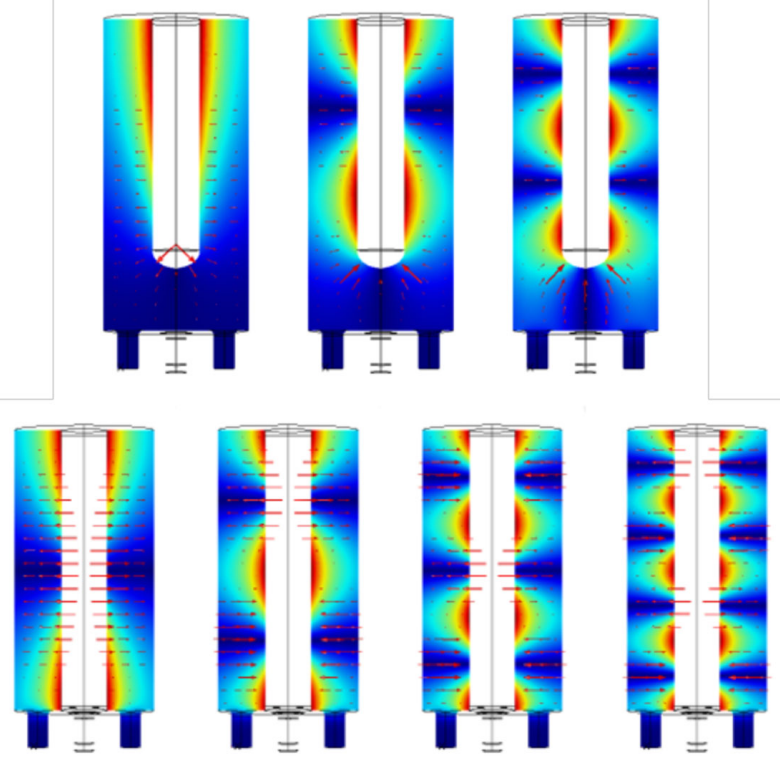

FIG. 2. Field distribution in the QWR (top) and HWR (bottom) in the investigated eigenmodes. The heat map shows the magnetic field and the arrows the electric field. and the sensitivity of the geometries to trapped magnetic flux, all as a function of rf frequency and different cavity treatments.

A critical part of understanding the SRF cavity performance is the temperature dependence of the surface resistance $R_{s}$, which can be expressed as

$$
R_{s}(T)=R_{T d}(T)+R_{T i},
$$

with $R_{T d}$ as the temperature-dependent term and $R_{T i}$ as the temperature-independent term. $R_{T d}$ can be calculated numerically based on the Mattis-Bardeen theory [24] and is approximated [25] as

$$
R_{T d}(T) \simeq \frac{\mu_{0}^{2} \omega^{2} \lambda^{3} \Delta}{\rho_{s} k_{B} T} \ln \left(\frac{C_{1} k_{B} T}{\hbar \omega}\right) \exp \left(\frac{-\Delta}{k_{B} T}\right)
$$

with $\omega$ as the resonance frequency, $\lambda$ as the Londonpenetration depth, $\Delta$ as the energy gap, $\rho_{s}$ as the normal state conductivity, $C_{1} \approx 9 / 2$, and $T$ as the temperature. Assuming that these parameters are not frequency dependent, Eq. (2) predicts a frequency dependence of $R_{T d} \propto \omega^{\sim 1.87}$.

One specific goal of the cavities presented in this paper is the determination of the frequency dependence of the surface resistance and an investigation of any deviations from theory. Previous research has been done with lead on copper cavities [26] at low power levels. Other studies have used several elliptical cavities of the same shape but different sizes [27]. Here, the challenge is to ensure that the surface and environmental conditions are comparable for the different test cavities.

Another approach is to use a sample cavity which can be excited at multiple frequencies such as the quadrupole resonator (QPR). The QPR has originally been designed for measurements at $400 \mathrm{MHz}$ [28]; it was later refurbished for multiple frequencies [29] and optimized by HelmholtzZentrum Berlin [30]. There are still open questions on how to translate results from the QPR to accelerating cavity performance.

A HWR-type cavity similar to the cavities described here has been developed at Center for Accelerator Science at Old Dominion University [31,32].

While Eq. (2) explicitly shows a frequency dependence of $R_{T d}$, one should note that the underlying Mattis-Bardeen theory is a zero-field limit theory. The field dependence of the overall surface resistance $R_{s}$ and its components is a topic of active research. Several models can describe a commonly observed increase of the surface resistance with an applied field. For example, pair-breaking [33], thermal feedback [34], and impurity scattering [35] models or the so-called percolation model [36] predict a $R_{s}\left(B_{p}\right) \propto B_{p}^{2}$ dependence, while other models, for example, a weak superconducting layer on top of the bulk material [37], suggest $R_{s}\left(B_{p}\right) \propto \exp B_{p}$ dependence. Another nonlinear model [38] tries to include the decreasing surface resistance 
with an increasing rf field, which is observed in nitrogendoped cavities [17].

\section{METHODOLOGY}

\section{A. Cavities}

The two cavities are used in a similar way as $1.3 \mathrm{GHz}$ single-cell cavities, as pure test cavities in a bath cryostat. To avoid perturbations of the TEM-mode field configuration, beam ports have been removed, and all rf ports have been moved to one end plate of the cavities. This is possible as the cavities will not be used for beam acceleration. Since the cavity will be submerged in liquid helium in a bath cryostat, a helium jacket is not necessary. A high shunt impedance and low surface field ratios were not design goals as they would be in an accelerating cavity. Instead, the design focused on achieving similar peak surface field ratios $E_{p} / B_{p}$ for all the relevant modes as well as the usage of common components such as identically dimensioned outer and inner conductors, identical rinse ports, and the same mechanical components and fixtures. One design limitation was imposed by the size of the induction furnace, which was designed for $1.3 \mathrm{GHz}$ single-cell cavities. This determined that the maximum outer dimensions of the cavities are restricted to a diameter of $200 \mathrm{~mm}$ and a length of $490 \mathrm{~mm}$. Based on these restrictions, the lowest frequency for the fundamental mode of the QWR was $153 \mathrm{MHz}$, regardless of the gap between the inner conductor and the bottom plate. A choice was made for the fundamental QWR frequency to be around $200 \mathrm{MHz}$ and for the HWR to be around $400 \mathrm{MHz}$.

A straight inner conductor (IC) was chosen to mitigate field distortion in HOMs and at the same time to simplify fabrication. This also allows for a moving $T$-mapping system to be inserted into the inner conductor. The diameters of the inner and outer conductor were chosen to be 60 and $180 \mathrm{~mm}$, respectively, matching the ISAC-II QWR cavities [39], allowing for reuse of forming dies. The top and bottom plates are flat, eliminating higher-level multipacting barriers and simplifying fabrication.

Further design choices were made to minimize the peak field ratio $E_{p} / B_{p}$ to push potential field emission onset to higher $B_{p}$ values.

For a HWR-type cavity of coaxial length $L$ with constant inner and outer conductor radii $a$ and $b$ and peak rf current and voltage of $I_{0}$ and $V_{T}$, respectively, the radial electric field $E_{r}$ and the tangential magnetic field $B_{\theta}$ at $a \leq r \leq b$ and $0 \leq z \leq L$ are given by

$$
\begin{gathered}
E_{r}=-j \frac{\eta I_{0}}{\pi r} \sin \left(\frac{p \pi z}{L}\right) e^{j \omega t}, \\
B_{\theta}=\frac{\mu_{0} I_{0}}{\pi r} \cos \left(\frac{p \pi z}{L}\right) e^{j \omega t},
\end{gathered}
$$

respectively, with $\omega=p \pi c / L$ with $p=1,2,3, \ldots$, and $\eta=\sqrt{\mu_{0} / \varepsilon_{0}}$. Using

$$
V_{T}=\eta \frac{I_{0}}{\pi} \ln \left(\frac{b}{a}\right),
$$

from Eqs. (3) and (4) follows that the peak fields $E_{p}$ and $B_{p}$ and their ratio are

$$
\begin{gathered}
E_{p}=\frac{V_{T}}{a} \frac{\pi}{\ln \left(\frac{a}{b}\right)}, \\
B_{p}=\frac{V_{T}}{a c} \frac{\pi}{\ln \left(\frac{a}{b}\right)}, \\
\Rightarrow \frac{E_{p}}{B_{p}}=c,
\end{gathered}
$$

respectively, with $c=1 / \sqrt{\varepsilon_{0} \mu_{0}}$ as the speed of light. Attention has to be made for the ports, as sharp edges in areas with high surface currents such as the end plates can enhance the magnetic fields, increasing the peak field ratio. The fillet radius at this edge was optimized to mitigate this field enhancement, resulting in no increase of the peak field ratio, as can be seen in Table I.

For a QWR-type cavity, the $E_{p} / B_{p}$ values are determined by the geometry of the IC tip and are, therefore, higher than the HWR values. Optimization of the QWR geometry focused on the IC tip cap, described by the ratio of vertical to horizontal size of the tip, and the capacitative gap, with the parameter space shown in Fig. 3. The optimization considered both the fundamental mode at $200 \mathrm{MHz}$ and the next-higher TEM mode at around $600 \mathrm{MHz}$ and is shown in Fig. 4. A peak field ratio of $0.47(\mathrm{MV} / \mathrm{m}) / \mathrm{mT}$ was reached.

In the final design, the QWR has a fundamental resonance frequency of $217 \mathrm{MHz}$, and the HWR has a fundamental resonance frequency of $389 \mathrm{MHz}$.

Both cavities are equipped with four cleaning ports for accessing the rf volume with a wand to high-pressure rinse (HPR) the cavity. All four ports are on the same flat plate. From each port, the water jet from a nozzle covers about $1 / 3$ of the cavity surface, providing sufficient overlap

TABLE I. The rf parameters of the two cavities for the TEM modes under investigation.

\begin{tabular}{lcccc}
\hline \hline \multicolumn{3}{c}{ Frequency $(\mathrm{MHz})$} & $\begin{array}{c}E_{p} / B_{p} \\
{[(\mathrm{MV} / \mathrm{m}) / \mathrm{mT}]}\end{array}$ & $U / B_{p}^{2}\left(\mathrm{~mJ} / \mathrm{mT}^{2}\right)$ \\
Cavity & 217 & 0.4796 & 0.4208 & $\mathrm{G}(\Omega)$ \\
\hline QWR & 648 & 0.4679 & 0.4350 & 113.7 \\
QWR & 389 & 0.2975 & 0.4675 & 60.39 \\
HWR & 778 & 0.2981 & 0.4681 & 120.77 \\
HWR & 1166 & 0.2981 & 0.4692 & 181.8 \\
HWR & 1555 & 0.2990 & 0.4708 & 241.24 \\
HWR & & & & \\
\hline \hline
\end{tabular}




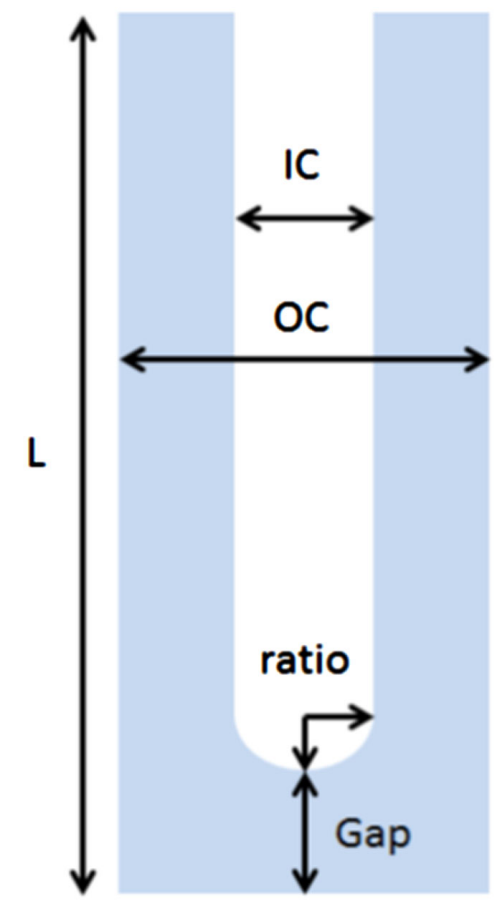

FIG. 3. The parametric simulation model of the QWR, in which the shaded area shows the rf space in the cavity. Optimization of the peak field ratio focused on the inner conductor tip ratio and gap.

between rinse ports to cover the whole cavity. To prevent rf losses on non-niobium parts, the rinse ports are $60 \mathrm{~mm}$ long.

The cavities are made from pure niobium to prevent contamination with foreign materials during heat treatments. High residual resistivity ratio $(>250)$ niobium is used on the main body, while the port flanges and QWR bottom plate are made from reactor-grade niobium. Since these components see minimal if any rf fields, the reactorgrade niobium can be used without any loss of performance to reduce fabrication costs. Each cavity is a single body with all parts electron-beam welded together without a removable bottom plate. This prevents the rf field from reaching any non-niobium surface, such as vacuum gaskets. Vacuum seals are realized with indium wire seals on the four ports. A wall thickness of $2 \mathrm{~mm}$ was chosen for the cylindrical components, while the top and bottom plates were $4 \mathrm{~mm}$ thick to provide mechanical stability. This gives enough material to allow for surface resets via chemical etching. As estimated, $0.7 \mathrm{~mm}$ can be taken off before the mechanical integrity becomes questionable. A surface reset requires that about $30 \mu \mathrm{m}$ are taken off from the surface. Accounting for the $120 \mu \mathrm{m}$ that are taken off right after fabrication, this leaves the capability to reset the surface on each cavity about 20 times.

Further details of the cavity design can be found in Ref. [40]. Resonant frequencies, as well as numerically calculated peak field ratios $E_{p} / B_{p}$ and geometric factors
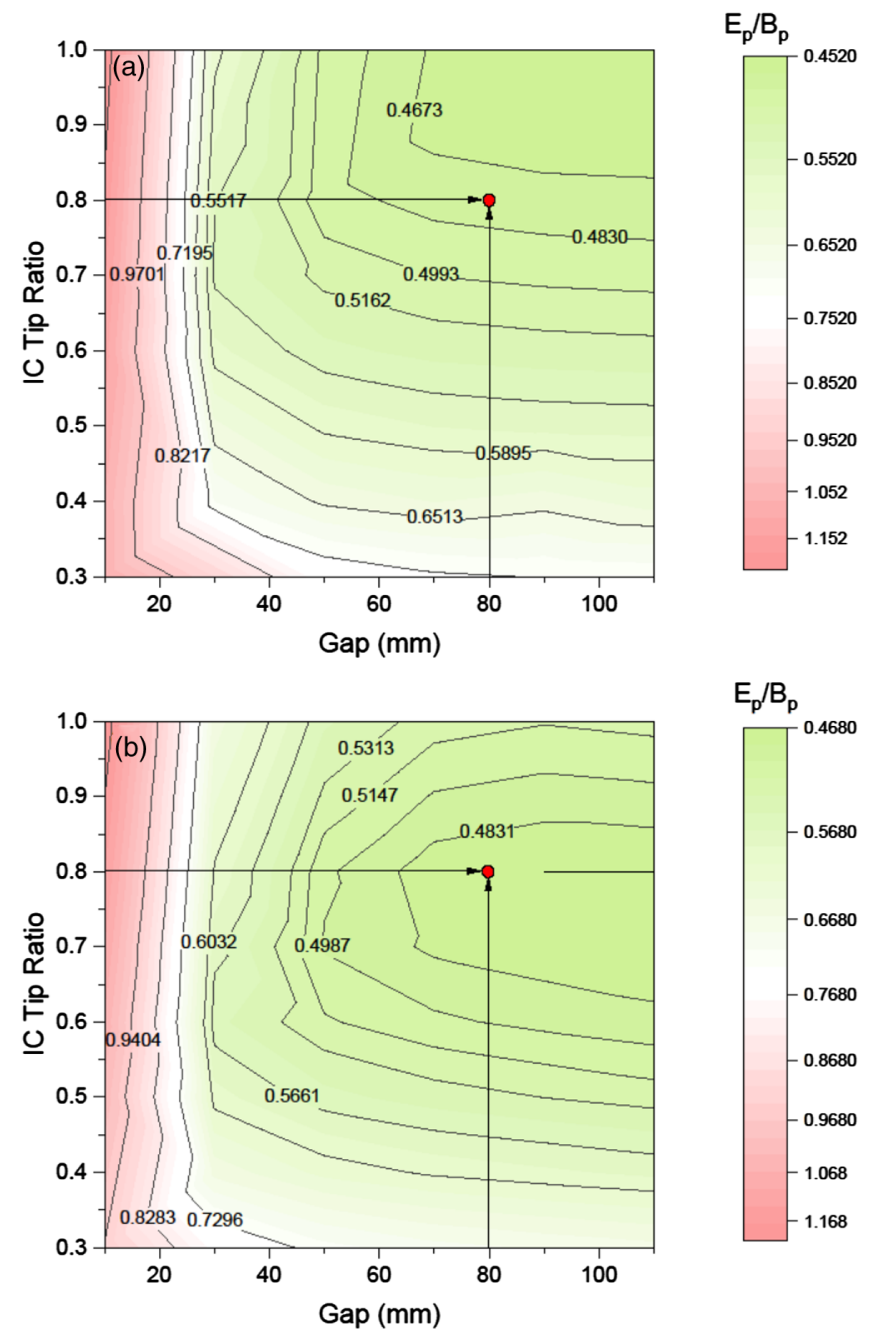

FIG. 4. Heat maps of $E_{p} / B_{p}$ as a function of the capacitive gap and IC tip ratio to determine a design with a similar $E_{p} / B_{p}$ in both the first (a) and second (b) TEM modes. The marker indicates the chosen design with $E_{p} / B_{p} \approx 0.47(\mathrm{MV} / \mathrm{m}) / \mathrm{mT}$.

$$
G=\omega \mu_{0} \frac{\int_{V}|\mathbf{H}|^{2} d V}{\int_{S}|\mathbf{H}|^{2} d S}
$$

with $\omega$ as the resonant frequency and $H$ as the magnetic field, for the TEM modes of interest are listed in Table I. The ratio of stored energy to magnetic peak field $U / B_{P}^{2}$ is fairly constant between the two cavities at $0.42 \mathrm{~mJ} / \mathrm{mT}^{2}$ for the QWR and $0.47 \mathrm{~mJ} / \mathrm{mT}^{2}$ for the HWR. Measured frequencies of the resonant TEM modes fall within $0.1 \%$ of the simulated values [41], giving confidence in the accuracy of the manufacturing to the specified dimensions.

\section{B. Available infrastructure}

A crucial part of the novel cavity treatments is hightemperature treatment at in the range from $100^{\circ}$ to $1000^{\circ} \mathrm{C}$ for a specified amount of time in either an ultrahigh-vacuum or low-pressure environment. For this, 
the TRIUMF induction furnace is used. To study the effects of external magnetic fields and flux expulsion, a set of 3D Helmholtz coils was designed and built around the cavities and existing cryostat. To control the cavities, the existing rf setup with some modifications is used. In this section, this infrastructure is described.

\section{Induction furnace}

For high-temperature heat treatments such as degassing [13], nitrogen doping [17], or nitrogen infusion [16], the TRIUMF induction furnace is used. The design is based on the JLab induction furnace [42] and dedicated to be used only for $\mathrm{Nb} \mathrm{SRF}$ cavities. In this furnace, a niobium susceptor is heated via rf induction. The heat generated in the susceptor is transferred to the cavity via radiation. Conventional ultrahigh-vacuum (UHV) furnaces pose a potential contamination risk, requiring the use of caps on the cavity ports [13]. In the induction furnace, the rf surface of the cavity has a line of sight to only $\mathrm{Nb}$ surfaces by design, reducing risk of contamination. In addition, slotted $\mathrm{Nb}$ caps (shown in Fig. 5) placed on the ports of the cavity provide an additional line of sight cover while allowing gas flow with a defined and reproducible leak between the UHV space and rf volume of the cavity. An advantage of the caps lies in the reduced effort to clean and refresh the surface via buffered chemical polishing (BCP), compared to removing and etching the susceptor of the furnace. A residual gas analyzer provides data during the degassing. A sample degassing spectrum during the $800{ }^{\circ} \mathrm{C}$ treatment of the HWR is shown in Fig. 6 along with the temperature profile.

\section{Helmholtz coils}

Flux trapping and the expulsion of external magnetic fields can be detrimental to the SRF cavity performance [43]. For example, the high- $Q_{0}$ performance of nitrogendoped cavities is very sensitive to external magnetic fields,

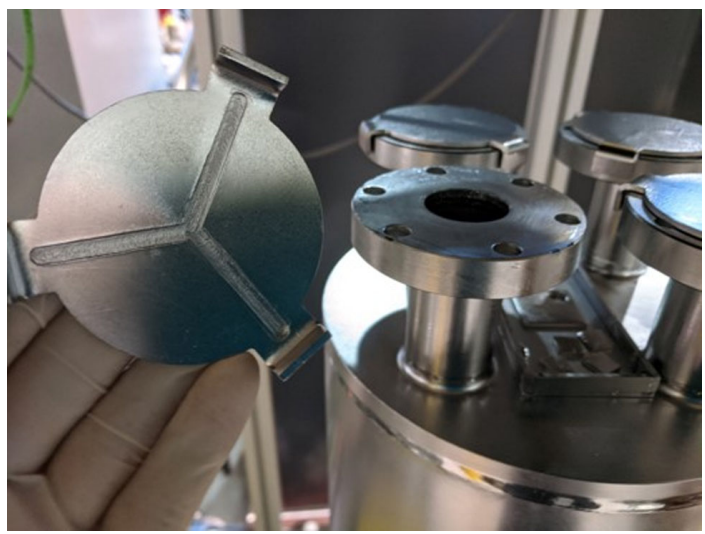

FIG. 5. Slotted Nb caps used to limit line of sight toward the rf surface while providing a defined and reproducible leak for gas flow from rf volume to the furnace volume.

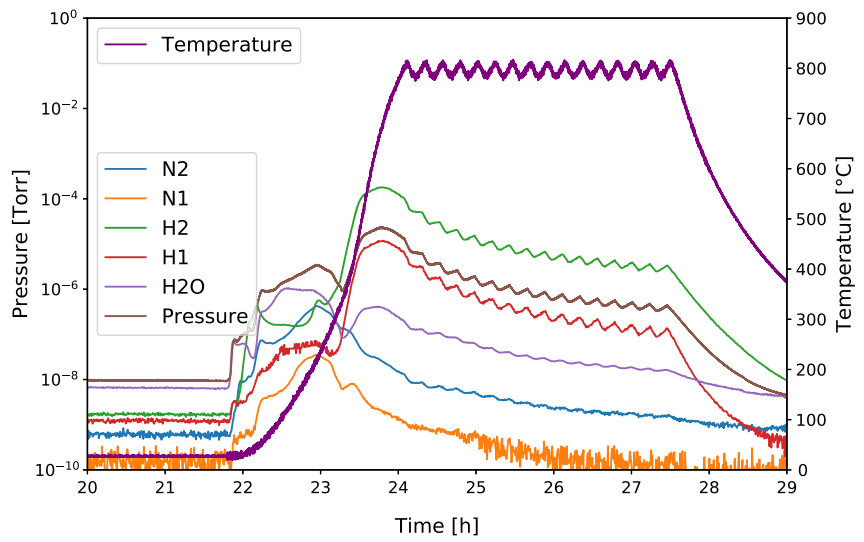

FIG. 6. Sample degassing treatment of the HWR at $800^{\circ} \mathrm{C}$. Shown are also partial pressures of gases of interest, showing a strong removal of hydrogen from the cavity.

so much that the specifications in the LCLS-II cryomodules calls for no more than $5 \mathrm{mG}$ of background field to preserve the high $Q_{0}$ of the cavities [44].

To control and manipulate the external magnetic field around the cavity in the TRIUMF cryostat, a set of three pairs of Helmholtz coils has been designed and built, shown in Fig. 7. These coils can be used to either cancel or control the external field to a specific value in all three spatial orientations. The current in each pair of coils can be controlled independently, to allow control of the direction of the field. One of the design criteria for the coils was a field uniformity greater than $95 \%$ over the cavity surface. To measure the magnetic field, three Bartington Mag F [45] cryogenic flux-gate probes are used. Magnetic field data as well as corresponding temperature data are collected via a Labview [46] program. This setup allows studies of how the performance of the coaxial cavities changes under different external magnetic field configurations and cooldown characteristics.
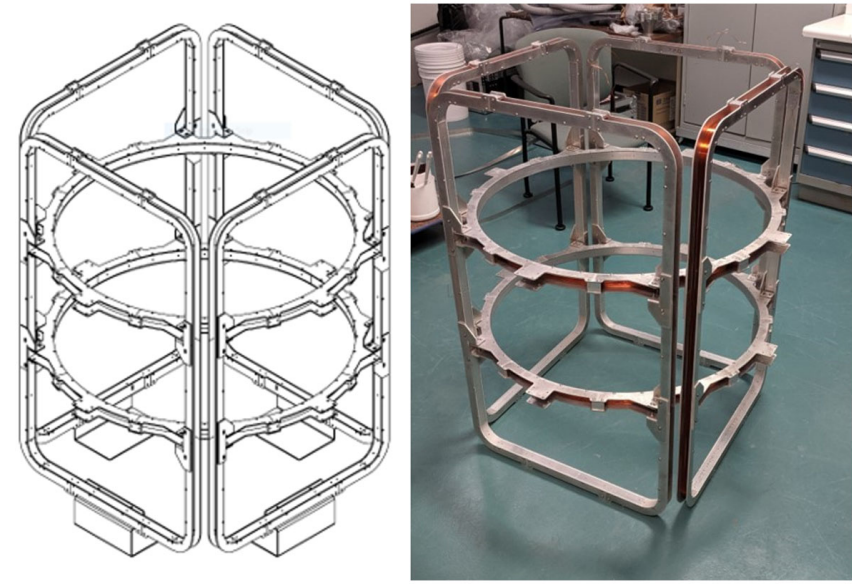

FIG. 7. Design (left) and realization (right) of the 3D Helmholtz coils before installation in the cryostat. 


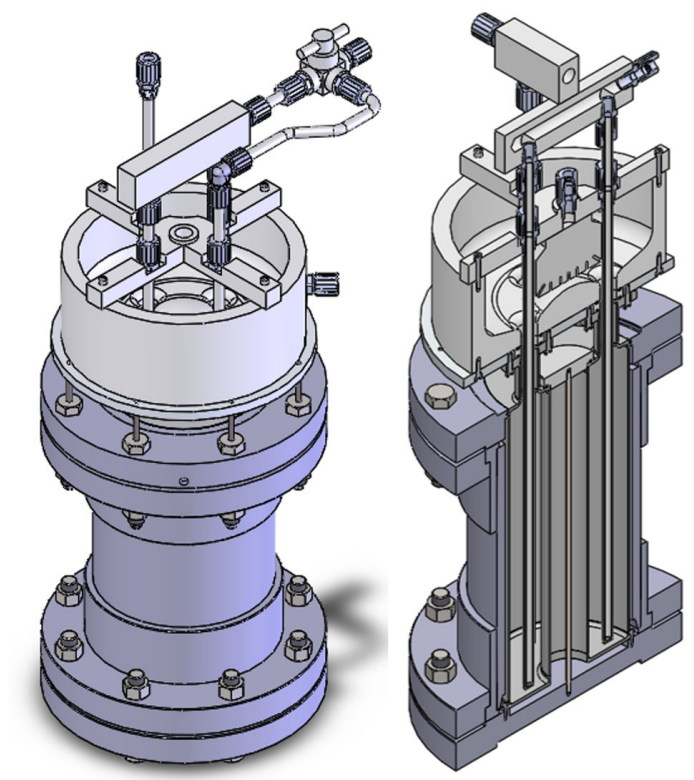

FIG. 8. Setup for BCP treatment with the water-cooling jacket. Acid is supplied to and pumped out from the bottom of the cavity via rods through the cavity ports.

\section{Chemical treatment and surface removal}

Chemical surface removal is done via BCP in a standard 1:1:2 mixture of hydrofluoric acid, nitric acid, and phosphoric acid. Figure 8 shows the design for the mechanical setup. Acid is supplied through a manifold and pumped to the bottom of the cavity via a diffuser, which prevents fast flowing jets of acid. An overflow reservoir at the top of the cavity ensures that all of the rf surface is in contact with the acid. From the reservoir, the acid flows back into the acid storage barrel, ensuring a constant flow of fresh acid through the cavity. The whole cavity is strapped into a water-cooling jacket to regulate the cavity temperature. The acid temperature in the storage barrel is controlled with a heat exchanger, which draws from the same cooling water. To cool the water, a $7 \mathrm{~kW}$ chiller from Advantage Engendering [47] is used. Water temperatures of between $10^{\circ} \mathrm{C}$ and $12^{\circ} \mathrm{C}$ are typically used. This results in etching rates of around $1 \mu \mathrm{m} / \mathrm{s}$. The manifold is also used to pump out the acid and supply the cavity with rinse water once the etch is done.

\section{4. $r f$ setup}

A self-excited loop (SEL) is utilized in the low-level rf (LLRF) control of cryostat cold tests at TRIUMF. The SEL frequency tracks the resonant frequency of the cavity. The frequency control is stable in either an open or closed amplitude loop and free of ponderomotive instabilities. The SEL, in the absence of phase loop feedback, is ideal for cavity performance characteristics, multipacting conditioning, and high-power pulse conditioning.

The LLRF boards developed for ISAC-II and Advanced Rare Isotope Experimental Laboratory e-linac projects [48] control at $140 \mathrm{MHz}$ and allow for either pulsed or continuous wave (cw) operation. An intermediate frequency is employed to down-convert the cavity frequency to $140 \mathrm{MHz}$ for input and to up-convert the output signal to the cavity resonant frequency for driving the rf amplifier. One essential part of the frequency converter is the high-performance bandpass filter. Discrete filters with $<-20 \mathrm{dBc}$ at $\pm 30 \mathrm{MHz}$ were chosen for 200 and $400 \mathrm{MHz}$, while cavity filters with $<-30 \mathrm{dBc}$ in the same range for the higher-frequency modes are used. The intermediate frequency and the bandpass filter are switched when changing test modes.

Two wideband solid-state amplifiers from BEXT [49] (70-650 MHz, $500 \mathrm{~W}$ ) and R\&K [50] (650-2800 MHz, $350 \mathrm{~W})$ are used to cover the frequency spectrum from $70 \mathrm{MHz}$ to $2.8 \mathrm{GHz}$ with up to $500 \mathrm{~W}$ of rf power.

Two variable rf couplers are used for the two different cavities: one based on an antenna coupler for the QWR to transfer power via the electric field and the other with a loop antenna for the HWR which couples to the magnetic field. To accurately measure $Q_{0}$, the coupler is moved to critical coupling with minimized reflected power. From a decay time measurement at low rf field, coupled with power and frequency measurements, $Q_{0}, Q_{p u}$, and $B_{p}$ are determined. This calibrates the setup for further measurement in continuous wave operation. The variable couplers allow measurement at or close to critical coupling over a wide range of $Q_{0}$, in the presented case up to 5 orders of magnitude. This is a significant advantage over a fixed coupler in terms of measurement accuracy [51], especially if a wide range of $Q_{0}$ is measured as is the case presented here. As a result, the measurement uncertainty is dominated by the directivity error of the directional coupler. This results in an apparent mismatch and a coupling constant away from unity, which leads to errors in the calibration constant that relates measured power to the field amplitude and propagates into the uncertainty of $Q_{0}$. Typical measurement uncertainties are $5 \%-10 \%$ for $Q_{0}$ and $2 \%-5 \%$ for $B_{p}$.

\section{Data preparation and fitting}

The data analysis presented here assumes that the surface resistance is uniform over the cavity surface. This is a generally accepted practice, but it is known that losses can vary locally [52], with hot spots several hundred $\mathrm{mK}$ warmer than the bath temperature.

Initial analysis is done by converting the quality factor data to the surface resistance $R_{s}^{*}$ through the well-known approximation

$$
R_{s}^{*}=G / Q
$$

where $G$ is the geometry factor as defined in Eq. (9). Field distributions and values for $G$ for all modes have been computed using COMSOL [53] and are given in Table I. 
Because of the nonuniform field distribution over the cavity surface and the field dependence of $Q_{0}$, the conversion $R_{s}^{*}=G / Q_{0}$ does not reveal the true field dependence of the surface resistance, and a correction has to be applied. This correction is especially important in TEMmode cavities, as the fields are significantly less uniform over the cavity surface compared to elliptical cavities. A variety of methods [36,54-56] can be used to extract the true surface resistance field dependence. In the methodology [57] adopted here, $R_{s}^{*}\left(B_{p}\right)$ data are first fitted with a power law series

$$
R_{s}^{*}\left(B_{P}\right)=\sum_{\alpha_{i}} r_{\alpha_{i}} B_{p}^{\alpha_{i}},
$$

with $r_{\alpha_{i}}$ as the fit parameter. $\alpha_{i}$ can be any non-negative real value and be chosen to best fit the data. The coefficients $r_{\alpha_{i}}$ then are corrected using parameters $\beta_{\alpha_{i}}$, which are derived from the field distribution over the surface of the cavity, resulting in the surface resistance

$$
R_{s}\left(B_{p}\right)=\sum_{\alpha_{i}} \beta\left(\alpha_{i}\right) r_{\alpha_{i}} B_{p}^{\alpha_{i}} .
$$

For a fairly uniform field distribution over the surface, such as in elliptical cavities, the factors $\beta\left(\alpha_{i}\right)$ are close to unity, while for TEM-mode cavities, these factors are significant larger than 1. Values for $\beta\left(\alpha_{i}\right)$ for the QWR and HWR modes have been calculated numerically, and a selection is given in Table II. Note that the HWR values are consistent for each mode, indicating that the field pattern is purely coaxial. The QWR values vary between modes due to the changing field pattern around the tip of the inner conductor. An example of the conversion from $Q_{0}$ to $R_{s}^{*}$ to $R_{s}$ at three different temperatures is shown in Fig. 9 for the $217 \mathrm{MHz}$ mode of the QWR.

To extract the temperature dependence of $R_{s}\left(B_{p}\right), Q_{0}$ is repeatedly measured during the cooldown from 4.2 to $2 \mathrm{~K}$ for a number of fixed peak field amplitudes $B_{p}$ in $10 \mathrm{mT}$ intervals up to a maximum field of $B_{\max }$. Each ramp up of the $\mathrm{rf}$ field up to $B_{\max }$ is considered as a set, measured roughly at the same temperature $T$ with differences of around $50 \mathrm{mK}$ between the first and last measurement points in each set. All sets are converted into $R_{s}^{*}$ using

TABLE II. $\beta\left(\alpha_{i}\right)$ values for the QWR and HWR investigated modes.

\begin{tabular}{lcccc}
\hline \hline$\beta_{i}$ & $\beta(0)$ & $\beta(1)$ & $\beta(2)$ & $\beta(3)$ \\
\hline QWR, 217 MHz & 1.0 & 1.432 & 1.778 & 2.061 \\
QWR, 648 MHz & 1.0 & 1.473 & 1.871 & 2.213 \\
HWR, 389 MHz & 1.0 & 1.463 & 1.857 & 2.193 \\
HWR, 778 MHz & 1.0 & 1.461 & 1.857 & 2.502 \\
HWR, 1166 MHz & 1.0 & 1.463 & 1.862 & 2.206 \\
HWR, 1555 MHz & 1.0 & 1.463 & 1.862 & 2.207 \\
\hline \hline
\end{tabular}
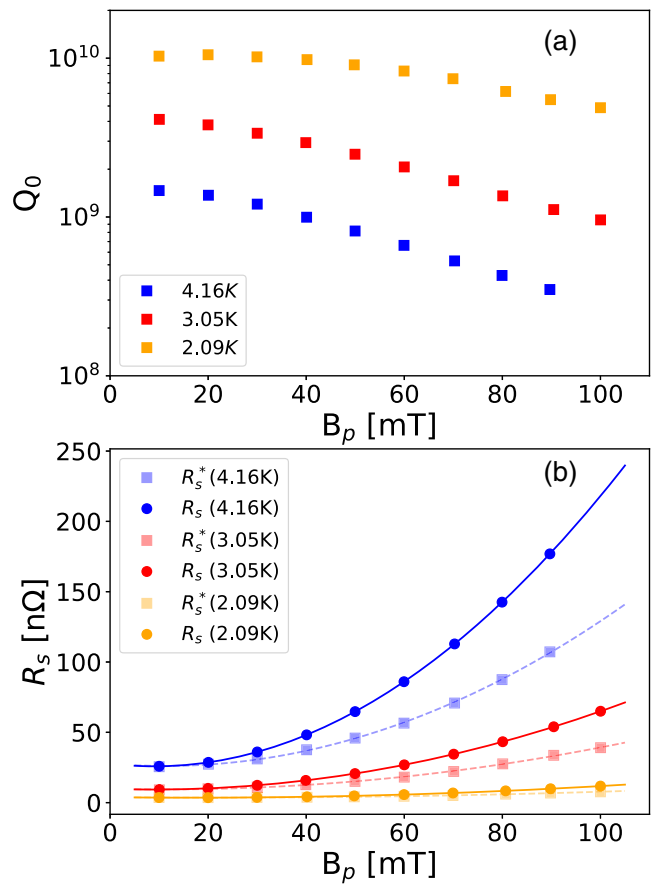

FIG. 9. $Q_{0}\left(B_{p}\right)$ for three different temperatures [markers in (a)] is first converted into $R_{s}^{*}$ [square markers in (b)] using Eq. (10). $R_{s}^{*}$ is then fitted to Eq. (11) [dashed lines in (b)], which then is corrected into $R_{s}$ [represented by the solid line and circular markers in (b)]. Error bars are omitted for clarity.

Eq. (10) and fitted to Eq. (11) to extract the parameters $r_{\alpha_{i}}$. In the investigated case, using a polynomial of third order was determined to be sufficient to describe the field dependence in the range of the available data accurately with very small residuals, well within measurement uncertainty. The parameters $r_{\alpha_{i}}$ are then multiplied by the corresponding $\beta_{i}$ to determine $R_{s}$ at the measured field and temperature. All sets are combined, sorted, and split by field amplitude to create new sets of $R_{S}(T)$.

In this analysis, it is assumed that the rf surface is isothermal and matches the bath temperature as measured by the installed temperature sensors, independent of dissipated rf power in the cavity walls. This is a reasonable approximation while the liquid helium is in the superfluid state below $2.17 \mathrm{~K}$ and at low $\mathrm{rf}$ power. At higher temperatures and rf powers, the reduced heat transfer between the cavity wall and the helium, due to effects like nucleate boiling and convective film flow variations, can cause local heating and increased surface resistance and, therefore, a temperature difference between measured bath temperature and $\mathrm{rf}$ surface temperature. This is observed in a small, but at higher fields noticeable, discontinuity of $R_{s}(T)$ at $2.17 \mathrm{~K}$. Further work is needed to fully model and account for this behavior for this particular set of cavities and rf resonant modes.

The extracted sets of $R_{S}(T)$ at fixed $B_{p}$ are fitted using the WinSuperFit [58] code v1.1 for each value of $B_{p}$ 
individually to a parametrized version of Eq. (2) in the form of

$$
\begin{aligned}
R_{s}(T) & =\frac{a_{0}}{T} \ln \left(\frac{4 k_{B} T}{\hbar \omega}\right) \exp \left(\frac{-a_{1}(T) T_{c}}{T}\right)+a_{2} \\
& =R_{T d}(T)+R_{T i}
\end{aligned}
$$

with $a_{0}, a_{1}(T)$, and $a_{2}$ as free fit parameters and $T_{c}=$ $9.25 \mathrm{~K}$ as the critical temperature. $a_{1}(T)$ represents the superconducting gap $\Delta$. The temperature dependence of $a_{1}$ is approximated as

$$
\frac{a_{1}(T)}{a_{1}(T=0 \mathrm{~K})}=\sqrt{1-\left(\frac{T}{T_{c}}\right)^{4}} .
$$

$R_{T d}$ and $R_{T i}$ are the extracted temperature-dependent and independent components of the surface resistance, respectively. Shifts in $\omega$ during the cooldown from 4.2 to $2 \mathrm{~K}$, which are primarily caused by the pressure and Lorentzforce detuning sensitivity of the cavity, are of small order compared to the frequency and are, therefore, ignored for the analysis. The error propagation is described in the Appendix. A collection of these $R_{S}(T)$ fits for the QWR $648 \mathrm{MHz}$ mode at fields up to $60 \mathrm{mT}$ is shown in Fig. 10. This is done for all measured modes to extract not only field dependence, but also frequency dependence of these fit parameters and the derived values of $R_{T d}$ at temperatures of interest. The quality of the fits is generally acceptable with $R^{2}$ values above 0.99 , producing fits well within the determined measurement uncertainty. At higher field

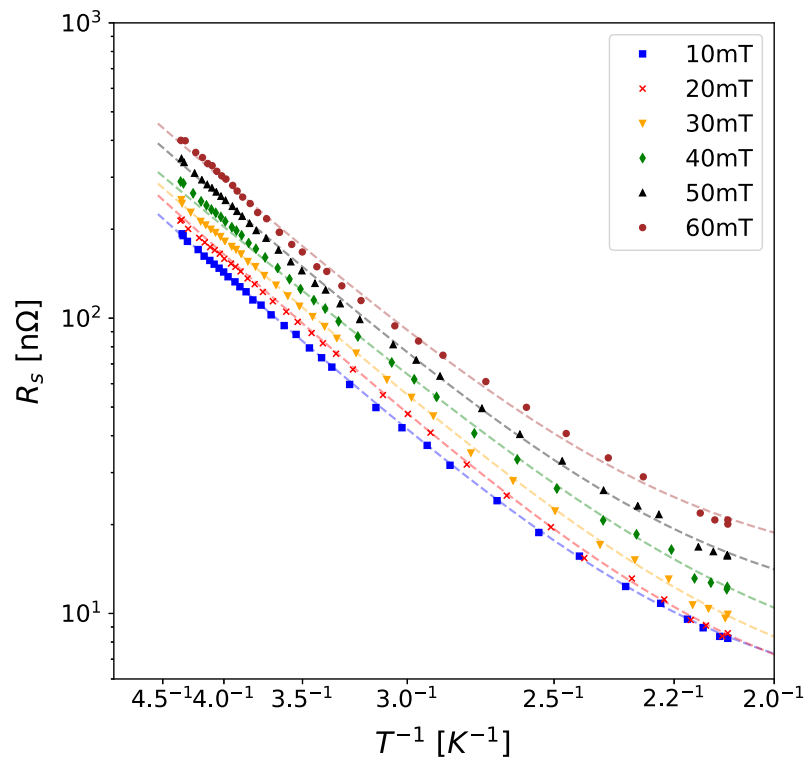

FIG. 10. Example of the $R_{s}$ fitting in the $648 \mathrm{MHz}$ mode with data in the range $B_{p}=10 \ldots 60 \mathrm{mT}$. The markers represent the measured data, while the dashed lines represent the fit of Eq. (13) to the data. Error bars are omitted for clarity. amplitudes, a distinct step in $R_{S}$ is observed at the $\lambda$ point of liquid helium of $2.17 \mathrm{~K}$. This is assumed to be an effect caused by a change in cooling capabilities between the normal and superfluid helium. A thorough analysis of this effect is in progress but beyond the scope of this paper.

\section{RESULTS}

\section{A. Cavity performance characterization}

The baseline surface treatment for both cavities presented in this paper includes a bulk surface removal via $\mathrm{BCP}$ of $120 \mu \mathrm{m}, 800^{\circ} \mathrm{C}$ degassing in the TRIUMF induction furnace $(6 \mathrm{~h}$ for the QWR, $3.5 \mathrm{~h}$ for the HWR; the difference is due to a larger hydrogen content in the QWR) to remove hydrogen from the cavities to prevent $Q$ disease, and a final $15 \mu \mathrm{m} \mathrm{BCP}$ surface etch to remove final contaminants. The cavity is then rinsed via HPR with ultrapure water, dried, and equipped with its pickup probe, variable coupler, and vacuum connections in a class 10 clean room environment. Initial measurements with the QWR were done in a horizontal orientation (with the coaxial axis horizontal). Subsequent QWR tests were done in a vertical orientation. All HWR tests were done in a vertical orientation. Once installed in the cryostat, the quality factor $Q_{0}$ as a function of peak surface field $B_{p}$ is characterized at 4.2 and $2 \mathrm{~K}$ at critical coupling with the movable coupler.

Combined QWR and HWR performance characterizations of the initial treatment are shown in Fig. 11 for $4 \mathrm{~K}$ and Fig. 12 for $2 \mathrm{~K}$. The presented data are for the uncorrected surface resistance $R_{s}^{*}$. The data for the two QWR modes were collected during a single cooldown of the QWR, the same as with the data for the three HWR

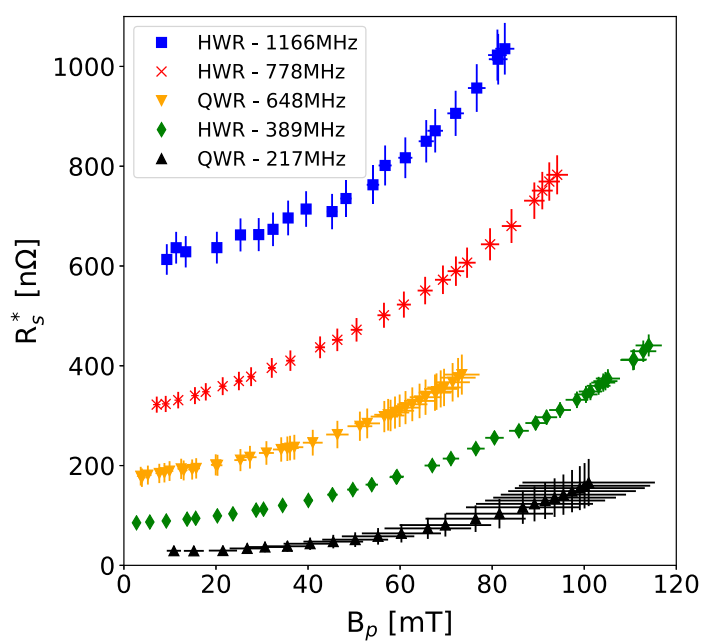

FIG. 11. Measured, uncorrected surface resistance $R_{s}^{*}\left(\propto 1 / Q_{0}\right)$ of the QWR and HWR at $4.2 \mathrm{~K}$ after degassing and $15 \mu \mathrm{m}$ surface removal. The measurement was free of detectable field emissions. The amplitude was limited by quench, except at $1166 \mathrm{MHz}$, where the amplifier power limit was reached. 


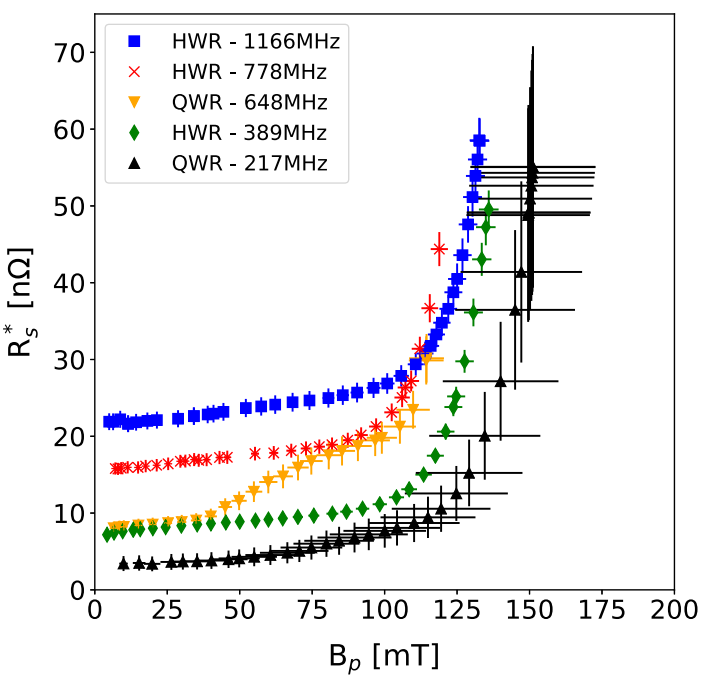

FIG. 12. Measured, uncorrected surface resistance $R_{s}^{*}$ of the QWR and HWR at $2.1 \mathrm{~K}$ after degassing and $15 \mu \mathrm{m}$ surface removal. The measurement was free of detectable field emissions.

modes. The larger error bars for the $217 \mathrm{MHz}$ mode compared to the other modes are due to a larger mismatch during the decay time measurement. Since then, procedural improvements have been made to reduce the uncertainty. No field emissions were observed during the measurements, indicating a cavity surface free from particulate contamination. For the presented data, the background field was compensated as close to zero as possible $(<1 \mu \mathrm{T})$ using the Helmholtz coils.

In the $4 \mathrm{~K}$ measurements, the surface resistance increases both with increasing field amplitude and with increasing frequency. The overall field dependence follows a similar behavior in all cavity modes. The field amplitude is limited by quench, except for the $1166 \mathrm{MHz}$ mode, which is limited by available amplifier power. The QWR has a reduced quench field compared to the HWR due to a different cavity orientation for the initial tests. The horizontal test orientation of the QWR reduced the liquid helium requirement in the dewar but produced early $\mathrm{cw}$ quenches at $4.2 \mathrm{~K}$ due to limited cooling and He-gas buildup in the inner conductor. Maximum quench field in the QWR was $100 \mathrm{mT}\left(E_{p}=47 \mathrm{MV} / \mathrm{m}\right)$. The HWR reached $115 \mathrm{mT}\left(E_{p}=35 \mathrm{MV} / \mathrm{m}\right)$.

At $2 \mathrm{~K}$, the average surface resistance is decreased significantly compared to the results at $4.2 \mathrm{~K}$, from hundreds of $\mathrm{n} \Omega$ to single-digit $\mathrm{n} \Omega$ in the lowest-frequency mode. In medium fields up to $100 \mathrm{mT}$, the field dependence of especially the HWR modes seems reduced significantly as well. In the QWR, features in the $R_{s}^{*}$ curve can be seen at around 60-75 mT, especially in the $648 \mathrm{MHz}$ mode. These could indicate insufficiently removed surface contamination after heat treatment. Above $100 \mathrm{mT}$ peak surface field, a strong increase in $R_{s}^{*}$ is measured without any measured field emission, which is characteristic of a high-field $Q$ slope [59]. The quench field was determined to be at $150 \mathrm{mT}\left(E_{P}=71 \mathrm{MV} / \mathrm{m}\right)$ for the QWR and $130 \mathrm{mT}$ $\left(E_{P}=40 \mathrm{MV} / \mathrm{m}\right)$ for the HWR.

In the following section, the results of the fielddistribution-corrected $R_{S}(T)$ fits are presented in terms of the temperature-dependent resistance $R_{T d}$ at 4.2 and $2.0 \mathrm{~K}$ and the temperature-independent resistance $R_{T i}$ based on Eq. (13). These components of $R_{S}$ are analyzed regarding their field and frequency dependence.

\section{B. Temperature-dependent surface resistance \\ 1. Field dependence}

Shown in Figs. 13 and 14 are calculated values for the temperature-dependent component $R_{T d}$ as a function of peak surface field $B_{p}$ at 4.2 and $2 \mathrm{~K}$, respectively, assuming an isothermal field-independent $\mathrm{rf}$ surface temperature as measured by the helium bath temperature. At both temperatures, an accelerated increase of $R_{T d}$ is observed as the rf field increases.

The two investigated field dependencies used to describe the increase of $R_{T d}$ are expressed as follows: a simple exponential growth

$$
R_{T d, e}\left(B_{p}\right)=R_{0, e} \exp \left(\gamma_{e} \frac{B_{p}}{B_{0}}\right)
$$

with $R_{0, e}$ as zero-field resistance, $\gamma_{e}$ as dimensionless growth rate parameter, and $B_{0}$ as normalizing parameter, which can be freely chosen; and a quadratic increase following

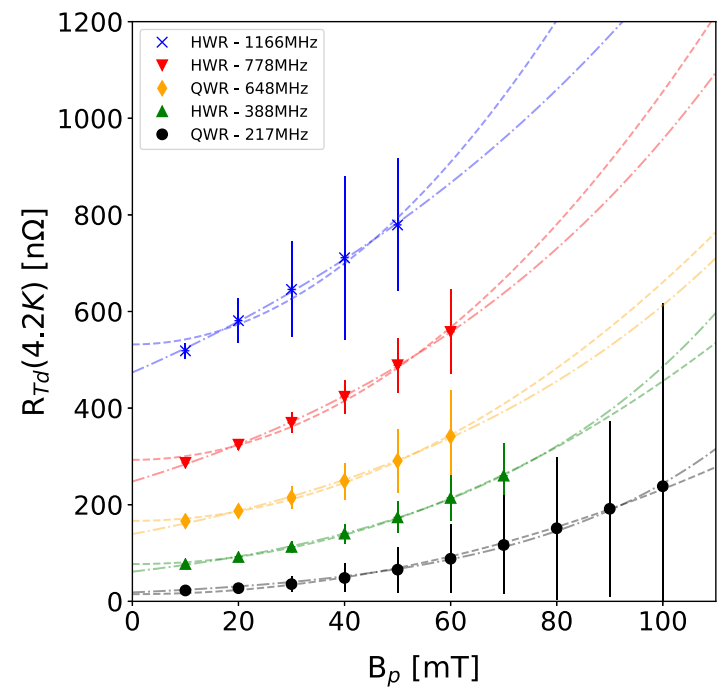

FIG. 13. $R_{T d}$ at a bath temperature of $4.2 \mathrm{~K}$ as a function of peak surface fields for five resonant modes, extracted from the cooldown data. Dashed lines represent fits to the exponential growth, while the dash-dotted lines show the quadratic increase. The data fit both trends well. 


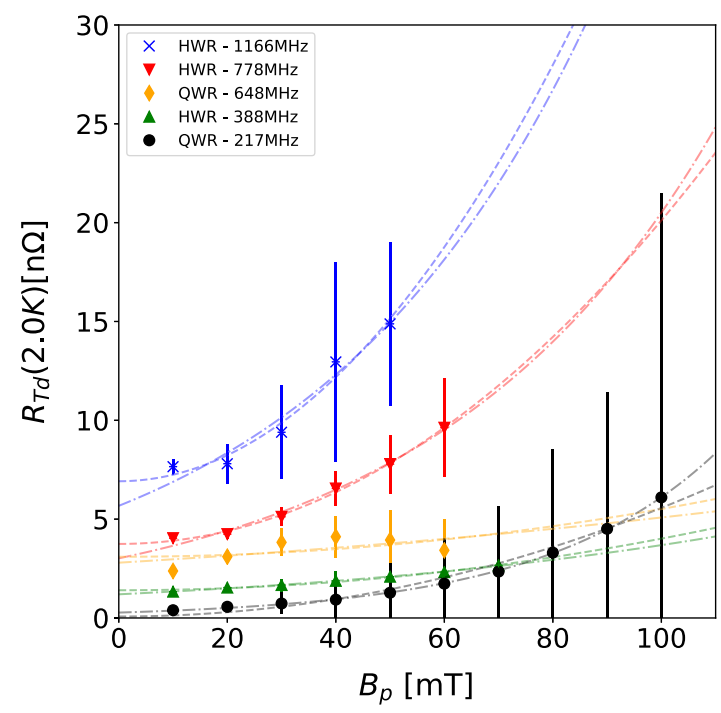

FIG. 14. $\quad R_{T d}$ at a bath temperature of $2 \mathrm{~K}$ shows a similar, but reduced field dependence compared to $R_{T d}(4.2 \mathrm{~K})$. Dashed lines represent fits to the exponential growth, while dash-dotted lines show the quadratic increase.

$$
R_{T d, q}\left(B_{p}\right)=R_{0, q}\left[1+\gamma_{q}\left(\frac{B_{p}}{B_{0}}\right)^{2}\right]
$$

with $R_{0, q}$ as zero-field resistance and $\gamma_{q}$ as the dimensionless slope parameter.

Within the determined uncertainty of $R_{T d}$, both Eqs. (16) and (17) describe the data fairly well as can be seen in Figs. 13 and 14 for bath temperatures of 4.2 and $2 \mathrm{~K}$, respectively, where dashed lines represent Eq. (16) while dash-dotted lines visualize Eq. (17). $R^{2}$ values for all fits are above 0.90, with most aggregating above 0.97 . Residual differences between the data and the two fit functions are generally of similar magnitude but slightly lower for the exponential fit. Both describe the data within the determined uncertainties. Thus, a definitive statement on the most appropriate field dependence cannot be made by the presented data alone. Supplemental measurements, for example, with material science probes such as the $\beta$-SRF beam line [60] at TRIUMF, would be needed to determine the physics behind the field dependence.

At a bath temperature of $2 \mathrm{~K}$, shown in Fig. $14, R_{T d}$ is, as expected, significantly lower than at $4.2 \mathrm{~K}$. This is unsurprisingly expressed in a lower zero-field resistance $R_{0}$. Both $\gamma_{e / q}$ parameters, on the other hand, do not show a clear trend in change between the two temperatures, indicating that the perceived reduced field dependence at a lower temperature is a result of the overall reduced magnitude of the zero-field resistance $R_{0, e / q}$. The fit results for all modes in both temperatures are listed in Table III.
TABLE III. Fit parameters for the field dependence of $R_{T d}$, Eqs. (16) and (17), with $B_{0}=100 \mathrm{mT}$. $R_{0, e / q}$ in $\mathrm{n} \Omega$.

\begin{tabular}{lccccc}
\hline \hline Mode & $T(\mathrm{~K})$ & $R_{0, e}$ & $\gamma_{e}$ & $R_{0, q}$ & $\gamma_{q}$ \\
\hline QWR, 217 MHz & 4.2 & $17.0(3)$ & $2.65(7)$ & $20.5(3)$ & $9.1(4)$ \\
QWR, 217 MHz & 2 & $0.292(4)$ & $3.01(5)$ & $0.354(9)$ & $11.9(9)$ \\
HWR, 389 MHz & 4.2 & $62.9(6)$ & $2.00(4)$ & $73.7(6)$ & $5.5(2)$ \\
HWR, 389 MHz & 2 & $1.19(1)$ & $1.14(4)$ & $1.32(2)$ & $2.4(3)$ \\
QWR, 648 MHz & 4.2 & $144(1)$ & $1.34(5)$ & $161(1)$ & $3.4(2)$ \\
QWR, 648 MHz & 2 & $2.1(1)$ & $1.5(4)$ & $2.4(1)$ & $4(2)$ \\
HWR, 778 MHz & 4.2 & $251.4(8)$ & $1.30(2)$ & $279(3)$ & $3.3(3)$ \\
HWR, 788 MHz & 2 & $3.5(1)$ & $1.4(2)$ & $3.86(6)$ & $3.8(4)$ \\
HWR, 1166 MHz & 4.2 & $467(2)$ & $1.05(2)$ & $509(6)$ & $2.5(4)$ \\
HWR, 1166 MHz & 2 & $6.6(4)$ & $1.3(4)$ & $3.7(2)$ & $3.6(8)$ \\
\hline \hline
\end{tabular}

\section{Frequency dependence}

Equation (2) predicts $R_{T d}$ to rise with increasing frequency according to $\omega^{1.87}$. To determine the frequency dependence, $R_{T d}$ is plotted as a function of the frequency for fields of up to $50 \mathrm{mT}$ as shown in Figs. 15 and 16 for 4.2 and $2 \mathrm{~K}$, respectively. Also shown are best fit lines in the form of

$$
R_{T d}(\omega)=A_{0} \omega^{x}
$$

with $A_{0}$ and $x$ as free fit parameters. Equation (18) will show up in the log-log plots as a straight line with a slope equal to the exponent $x$. Based on the fit lines in Figs. 15 and 16, the exponent $x$ seems to have a field dependence. Figure 17 shows $x$ as a function of the rf field for both

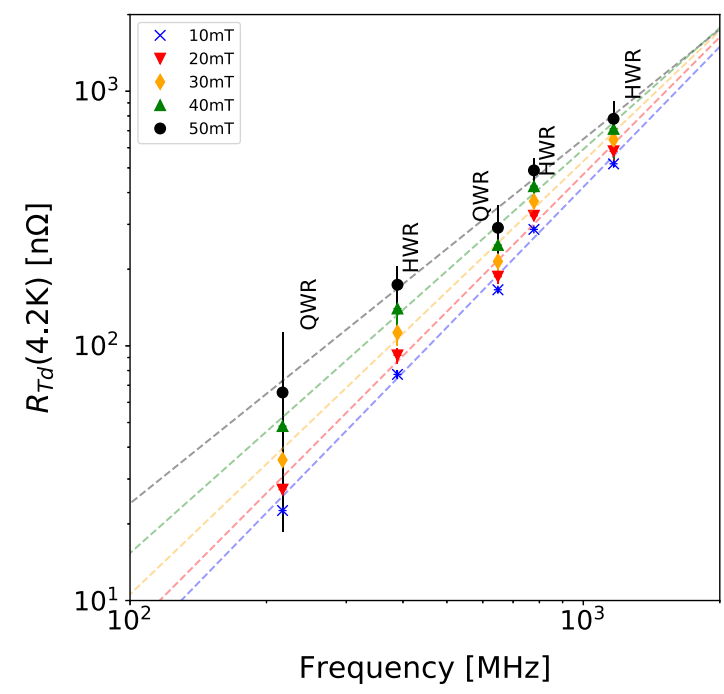

FIG. 15. Combined $R_{T d}$ data from QWR and HWR tests at $4.2 \mathrm{~K}$ as a function of the frequency for rf fields up to $50 \mathrm{mT}$. Dashed lines show best fits of Eq. (18) to the data and indicate a decrease of the frequency dependence with increasing rf field. 


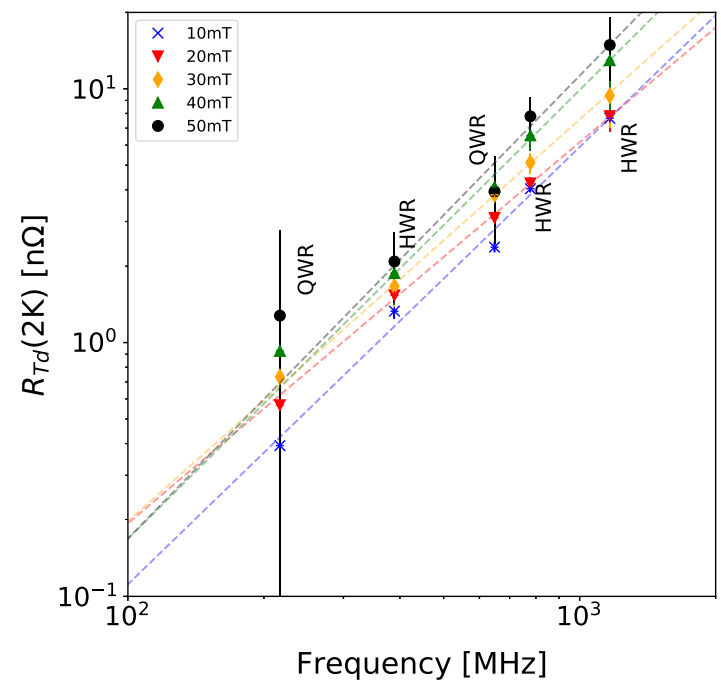

FIG. 16. Combined $R_{T d}$ data from QWR and HWR tests $R_{T d}$ at $2 \mathrm{~K}$ as a function of the frequency. Dashed lines indicate best fits of Eq. (18) to the data. A similar trend as at $4.2 \mathrm{~K}$ of a decreasing frequency dependence is observed.

temperatures. At low field and $4.2 \mathrm{~K}$, the exponent is determined as $1.8(2)$, which matches with the predicted value of 1.87 . At $2 \mathrm{~K}$ and low field, the exponent is lowered to $1.7(2)$, also matching the predicted value within the determined uncertainty. There seem to be opposing trends between $x$ at 4.2 and $2 \mathrm{~K}$. At $4.2 \mathrm{~K}$, it is trending down, but at $2 \mathrm{~K}$ it increases. But both trends have significant uncertainty, making a conclusion challenging. Examination of this trend is subject to further studies.

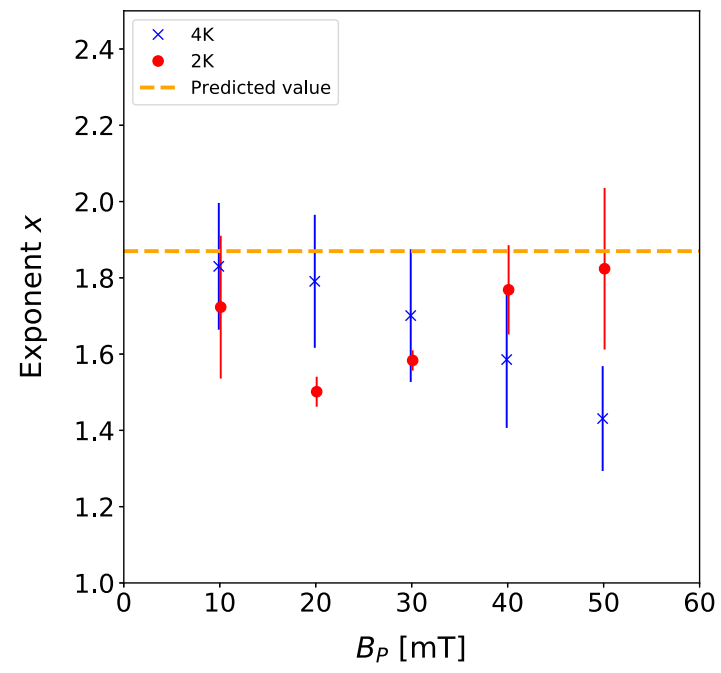

FIG. 17. The exponent $x$ of the frequency dependence, Eq. (18), matches the prediction, $x \approx 1.87$, by the theoretical model, Eq. (2) (dashed line) within the determined uncertainties at low fields at both 4.2 (square markers) and $2 \mathrm{~K}$ (circular markers) but trends toward lower values at higher field, deviating from the prediction.

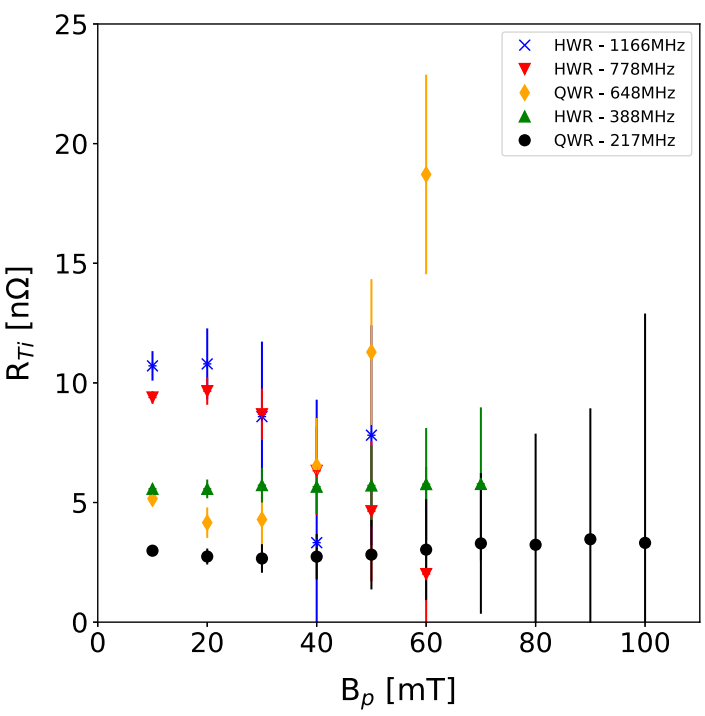

FIG. 18. Combined QWR and HWR $R_{T i}\left(B_{p}\right)$ data for all investigated modes. No consistent trend between the modes can be determined. The sharp increase in the $648 \mathrm{MHz}$ modes at $B_{p}>40 \mathrm{mT}$ is attributed to insufficient contaminant removal in a high field area of this mode.

\section{Temperature-independent resistance}

Figures 18 and 19 show the field and frequency dependency of $R_{T i}$, respectively. The sharp increase of $R_{T i}$ at $648 \mathrm{MHz}$ at fields of $40 \mathrm{mT}$ and higher may be attributed to insufficient removal of contaminants after the heat treatment. Since this increase is not observed in the $217 \mathrm{MHz}$ mode, this may indicate contaminants in a region of high fields for the second QWR mode but relatively low fields for the first QWR mode. This would mean a nonuniform distribution of the surface resistance in this

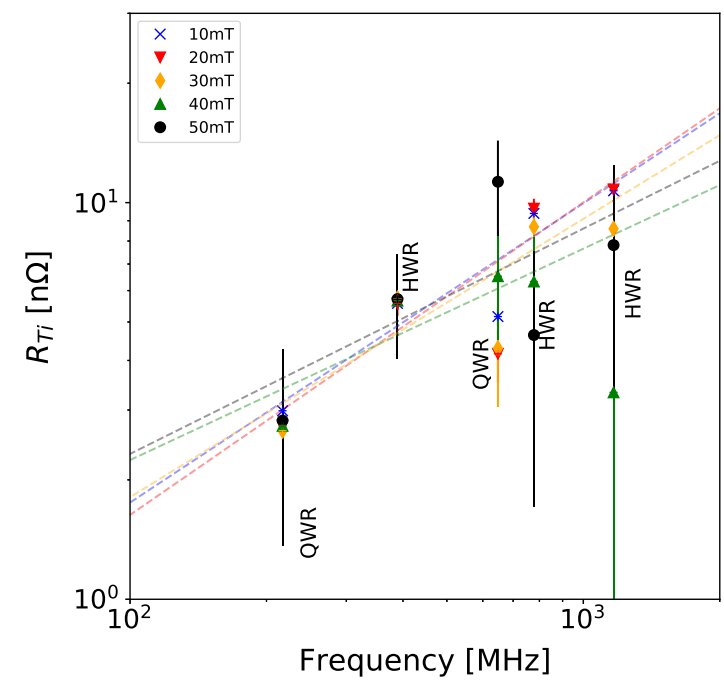

FIG. 19. Combined QWR and HWR data for $R_{T i}(\omega)$ reveal an increasing trend with increasing frequency for all field amplitudes, although there is a large scatter in the data. 
particular case. Otherwise, no clear field dependence can be identified. Regarding the frequency dependence, an overall increasing trend is extracted out of the cooldown data. Averaged over the measured rf field amplitudes, $R_{T i}$ is $\propto \omega^{0.7(1)}$. This matches the frequency dependence of normal conducting losses in the anomalous limit of $\omega^{2 / 3}$.

\section{QWR $120^{\circ} \mathrm{C}$ baking}

A common cavity preparation is $120^{\circ} \mathrm{C}$ baking for $48 \mathrm{~h}$. In the presented case, the baking is done with resistive heaters strapped to the cavity, while the cavity is installed in the cryostat. During the bake, both sides of the cavity wall, rf space and helium space surrounding the cavity, are under vacuum. The effect of this bake on $R_{s}^{*}$ of the QWR is shown in Figs. 20 and 21 for 4.2 and $2 \mathrm{~K}$, respectively. A clear decrease in both the amplitude and field dependence of $R_{S}^{*}$ is shown at $4.2 \mathrm{~K}$, while at $2 \mathrm{~K}$ a slight increase in $R_{s}^{*}$ is visible. A conclusion can be made that the $120^{\circ} \mathrm{C} / 48 \mathrm{~h}$ treatment reduces $R_{T d}$, which dominates at $4.2 \mathrm{~K}$, while slightly increasing $R_{T i}$. The reduction of $R_{T d}$ at $2 \mathrm{~K}$ is insignificant compared to the increase of $R_{T i}$. This is consistent with previously observed behavior in BCPed coaxial cavities [21]. In elliptical $1.3 \mathrm{GHz}$ cavities, an improvement in $Q_{0}$ is seen even at $2 \mathrm{~K}$ [61] due to the strong frequency dependence of $R_{T d}$.

At the time of writing, the HWR is in preparation for this surface treatment, and, once completed, a full analysis with frequency dependence will be done.

\section{E. Helmholtz coil demonstration}

The capabilities of the Helmholtz coils were demonstrated with the QWR. The cavity was first cooled down in a fully compensated external field, with the current in all

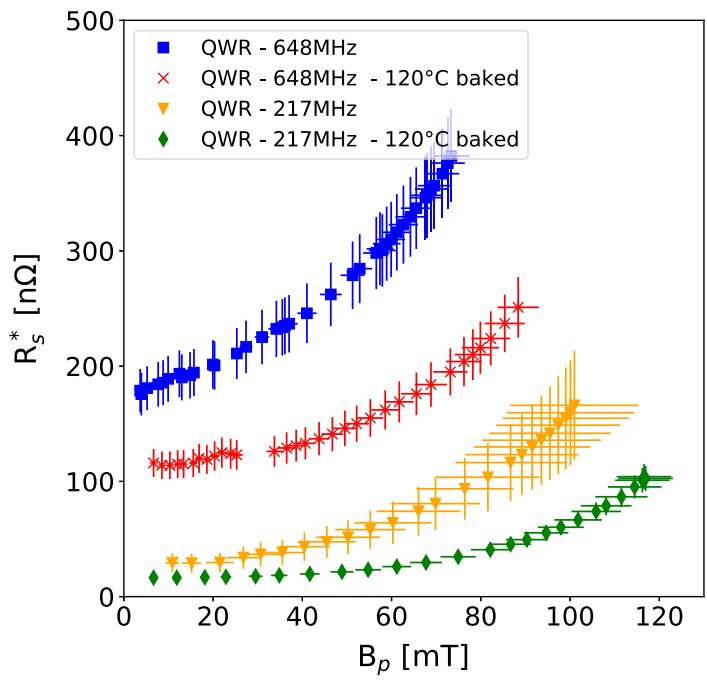

FIG. 20. Baking the QWR at $120^{\circ} \mathrm{C}$ for $48 \mathrm{~h}$ significantly reduces the uncorrected surface resistance $R_{s}^{*}$ at $4 \mathrm{~K}$ in both modes.

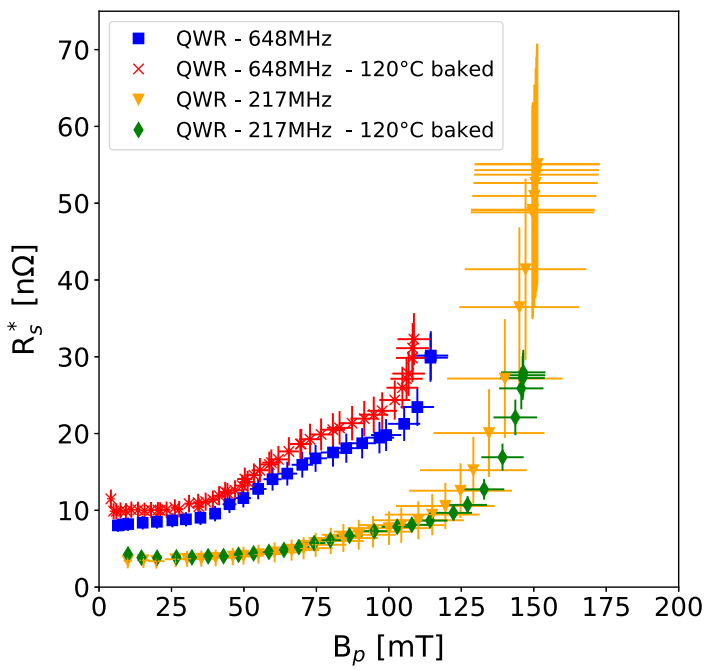

FIG. 21. At $2 \mathrm{~K}$, the $120^{\circ} \mathrm{C}$ bake increases $R_{s}^{*}$ marginally for both QWR modes.

coils tuned to an external field in all three spatial dimensions of $<0.5 \mu \mathrm{T}$. After characterization, the cavity was warmed up above transition to around $20 \mathrm{~K}$, the vertical coils tuned to $10 \mu \mathrm{T}$ at the geometric center of both the cavity and coils, and then cooled down again below transition. This thermal cycle has been repeated with a field of $20 \mu \mathrm{T}$ also in the vertical direction. The resulting surface resistance $R_{S}^{*}$ as a function of the peak surface field is shown in Figs. 22 and 23 for the 217 and $648 \mathrm{MHz}$ modes of the QWR at around $2.1 \mathrm{~K}$. The slopes in the medium field range up to $80 \mathrm{mT}$ are identical between the different external fields but do have an offset to each other, suggesting a constant addition to the surface resistance

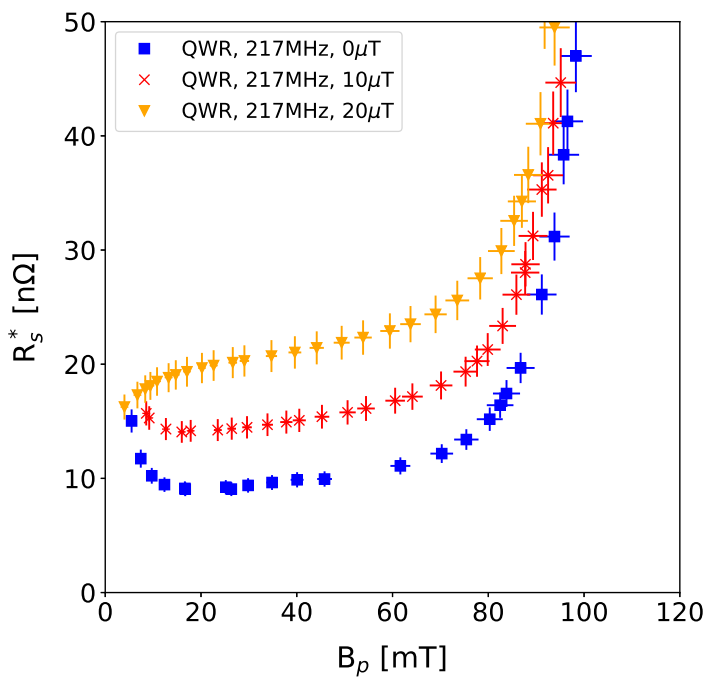

FIG. 22. $\quad R_{s}^{*}$ data of the $217 \mathrm{MHz}$ mode measured at $2.1 \mathrm{~K}$ with different dc external magnetic fields, aligned with the vertical cavity axis, shows a constant increase with the increased external field. 


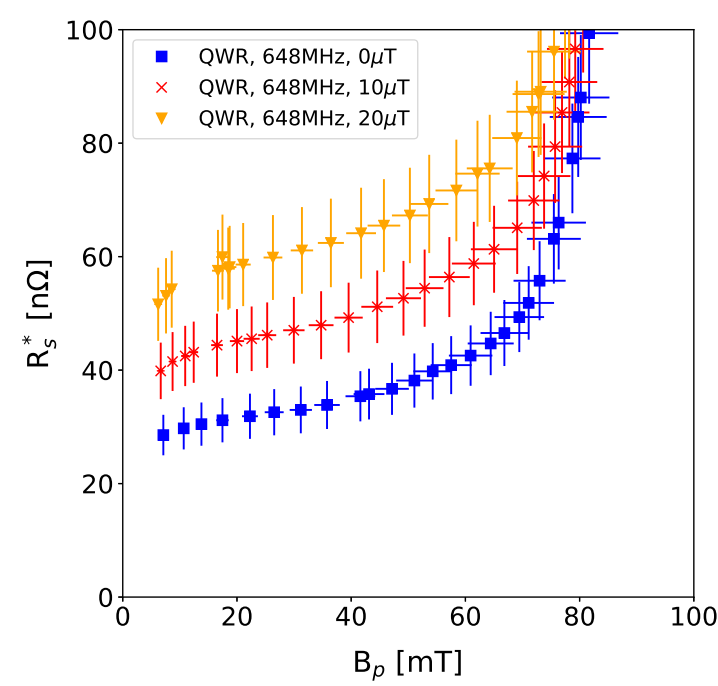

FIG. 23. At $648 \mathrm{MHz}$, a similar behavior compared to the $217 \mathrm{MHz}$ mode is recorded when changing the external dc magnetic field between thermal cycles.

caused by the external field. This amounts to a sensitivity $S$ of $\sim 0.5 \mathrm{n} \Omega / \mu \mathrm{T}$ at $217 \mathrm{MHz}$ and $\sim 1.5 \mathrm{n} \Omega / \mu \mathrm{T}$ at $648 \mathrm{MHz}$.

In Ref. [62], the magnetic field sensitivity $S$ assuming full flux trapping spread uniformly over the surface is specified in Eq. (9.5) as

$$
S=3 \frac{\mathrm{n} \Omega}{\mu \mathrm{T}} \sqrt{f}
$$

with $f$ as the resonant frequency in gigahertz. Using this, a sensitivity of around 1.4 and $2.4 \mathrm{n} \Omega / \mu \mathrm{T}$ at 217 and $648 \mathrm{MHz}$, respectively, would be expected. The difference between textbook and measured value suggests that either not all the field is trapped in the cavity walls or the flux is trapped in locations that do not contribute strongly to the surface resistance. Those would be areas with low magnetic surface fields, like the tip of the inner conductor, as these areas contribute to the losses significantly less than high field areas. It is important to note that the cavity does not cool down uniformly and different parts of the cavity transition into the Meissner state at different times, effectively changing the local magnetic field distribution as the cavity cools down [23]. A large thermal gradient across the cavity and a fast cooldown through the superconducting transition have a positive effect on flux expulsion, and less flux is trapped, leading to lower $R_{s}[18,63]$. Other models estimate the magnetic field sensitivity as a function of the geometry [22]. Studies to investigate the effect of cooldown and external fields will continue at TRIUMF using the QWR and HWR in combination with the Helmholtz coils and simulations.

At $4.2 \mathrm{~K}$, the additional surface resistance is too small to be significant. Following similar measurements with the HWR, a full analysis including frequency dependence will be done.

Further studies are needed and planned to explore the role of trapped magnetic flux in TEM-mode cavities and specific techniques to mitigate reduced performance due to flux trapping.

\section{SUMMARY}

The TRIUMF multimode coaxial SRF cavities are an excellent tool to study TEM-mode cavities. In particular, the dependence of the surface resistance on temperature, surface treatment, rf frequency, external magnetic field, and rf field amplitude are available to study, opening an unprecedented parameter space to be explored. The presented infrastructure in place at TRIUMF allows for exploration of this large parameter space. rf amplitudes of up to $150 \mathrm{mT}$ peak surface magnetic field have been reached. In the presented data, some early conclusions are drawn on the field and frequency dependence.

Characterization of both the QWR and HWR after degassing at $800{ }^{\circ} \mathrm{C}$ and a flash BCP surface removal show excellent performance at both 4.2 and $2 \mathrm{~K}$, on par with performances of $1.3 \mathrm{GHz}$ single-cell elliptical cavities with the same surface treatment. The cavities show a low surface resistance and high quench field.

Data collected during the cooldown at several rf field amplitudes and multiple resonant modes allow one to separate $R_{s}$ into its components $R_{T d}\left(T, B_{p}, \omega\right)$ and $R_{T i}\left(B_{p}, \omega\right)$ and analyze the frequency and field dependence of these parameters.

The data reveal that the temperature-dependent term $R_{T d}$ at low rf fields is $\propto \omega^{1.8(2)}$ at $4.2 \mathrm{~K}$ and $\propto \omega^{1.7(2)}$ at $2 \mathrm{~K}$, matching with the predicted dependence of $\omega^{1.87}$. The $\mathrm{rf}$ field dependence of $R_{T d}$ matches both a quadratic and an exponential growth model in the investigated range of field amplitudes. The change in slope between 4.2 and $2 \mathrm{~K}$ is dominated by the reduction of the zero-field resistance $R_{0}$ rather than the slope parameter $\gamma$.

The temperature-independent component $R_{T i}$ gives a less clear picture due to a large scatter in the data. An overall increasing trend with increasing frequency $\propto \omega^{0.7(1)}$ is found, which matches with anomalous losses. No clear conclusion can be drawn on the rf field dependence.

Capabilities to bake the QWR at $120^{\circ} \mathrm{C}$ have also been demonstrated, which resulted in a significant higher $Q_{0}$ at $4.2 \mathrm{~K}$ and a small decrease in $Q_{0}$ at $2 \mathrm{~K}$. This is attributed to a strong decrease in $R_{T d}$, which is the dominant term at $4.2 \mathrm{~K}$, and a small increase in $R_{T i}$, which is of comparable order to $R_{T d}$ at $2 \mathrm{~K}$. Since many low-frequency cavities installed in accelerators operate at $4.2 \mathrm{~K}$, the $120^{\circ} \mathrm{C} / 48 \mathrm{~h}$ bake is a very useful treatment to increase cavity performance.

The functionality of the Helmholtz coils has been demonstrated, and a first estimation of the external magnetic field sensitivity for a vertical field orientation measures a sensitivity of the QWR of $\simeq 0.5 \mathrm{n} \Omega / \mu \mathrm{T}$ at $217 \mathrm{MHz}$ and $\simeq 1.5 \mathrm{n} \Omega / \mu \mathrm{T}$ at $648 \mathrm{MHz}$. Just as in the elliptical cavity developments as the surface resistance is reduced by customized treatments, the performance impact of trapped 
flux in TEM cavities will become increasingly important especially in cases where the cavities will operate at $2 \mathrm{~K}$. Further refinement of the knowledge of flux expulsion in TEM cavities will be an important subject of development.

\section{A. Future work}

This paper shows the possibilities of the research areas covered by the coaxial multimode cavities at TRIUMF with examples of early performance measurement results. Future work will include comprehensive studies of the effects of various surface treatments, as well as changes of the background magnetic field, on the surface resistance. A further step in data preparation will also include corrections between the measured helium bath temperature and the rf surface temperature of the cavity. To fully understand the underlying physics of new treatments, sample measurements of small-scale witness samples are being done using facilities such as $\mu \mathrm{SR}[64,65], \beta$-NQR, and the upcoming $\beta$-SRF facility [60] as well as surface and material analysis tools such as SEM, TEM, and SIMS to correlate rf performance to fundamental parameters and material characteristics.

This work hopefully inspires other labs to acquire identical test cavities so that a global comparison between results would be possible.

\section{ACKNOWLEDGEMENTS}

The authors thank the technical team of the SRF group, namely, Devon Lang, James Kier, Ben Matheson, Ruminder Sekhon, and Bhalwinder Waraich, and cryogenics group, namely, Johnson Cheung and David Kishi, who helped tremendously with the collecting the data. This work was supported by the Natural Sciences and Engineering Research Council of Canada (NSERC) through Grants No. SAPIN-2019-00040, No. SAPPJ2017-00035, and No. SAPEQ-2015-00005.

\section{APPENDIX: ERROR PROPAGATION}

The measurement error in both $Q_{0}$ and $B_{p}$ determines the uncertainty in $R_{S}$. Following conventional error propagation rules,

$$
\frac{\delta R_{s}^{*}}{R_{s}^{*}}=\frac{\delta Q_{0}}{Q_{0}},
$$

as $G$ in Eq. (10) is considered a constant. This uncertainty in $R_{s}^{*}$ is used to weight the data points in the fits of $R_{s}^{*}\left(B_{p}\right)$ to Eq. (11), which determine $r_{\alpha_{i}}$, with $\delta r_{\alpha_{i}}$ determined by the $95 \%$ confidence interval of the fit. These fit uncertainties as well as $\delta B_{p}$ propagate into the calculation of $\delta R_{s}$ according to

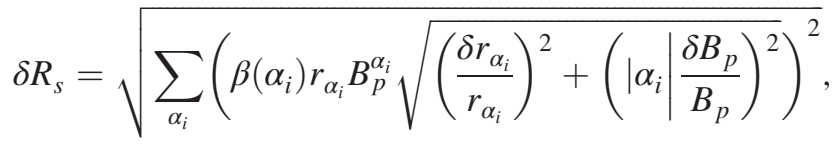

in which $\beta\left(\alpha_{i}\right)$ is assumed as without error, assuming sufficient care is done in the calculation [55]. The uncertainties in $R_{s}$ are used in the $R_{s}(T)$ fits to Eq. (13) which produce uncertainties $\delta a_{0}, \delta a_{1}$, and $\delta a_{2}$ for the fit parameters. $\delta R_{T i}$ is directly equal to $\delta a_{2}$, while $\delta R_{T d}$ is calculated using

$$
\frac{\delta R_{T d}}{R_{T d}}=\sqrt{\left(\frac{\delta a_{0}}{a_{0}}\right)^{2}+\left(\delta a_{1} \sqrt{\left.1-\left(\frac{T}{T_{c}}\right)^{4} \frac{T_{c}}{T}\right)^{2}} .\right.}
$$

Uncertainties for further fits for field or frequency dependence are done in Origin 2020 [66], which takes into account uncertainties and provides uncertainties for the fit parameters and fit quality measurements.

[1] J. J. Dilling, R. Krücken, and G. Ball, ISAC overview, Hyperfine Interact. 225, 1 (2014).

[2] M. Leitner et al., The FRIB project at MSU, in Proceedings of the International Conference on RF Superconductivity (SRF'13), Paris, France, 2013 (JACoW Publishing, Geneva, Switzerland, 2013), MOIOA01.

[3] D. Jeon, Status of the RISP superconducting heavy hon accelerator, in Proceedings of the International Conference on RF Superconductivity (SRF2015), Whistler, $B C, 2015$ (JACoW Publishing, Geneva, Switzerland, 2015), MOAA05, pp. 31-33.

[4] V. Lebedev (PIP-II Collaboration), The PIP-II reference design report, Technical Report, https://doi.org/10.2172/ 1365571.

[5] S. Peggs (ESS Collaboration), ESS conceptual design report, Technical Report, https://europeanspallationsource .se/sites/default/files/downloads/2017/09/CDR_final_120206 .pdf.

[6] F. Schlander, C. Darve, N. Elias, M. Lindroos, C. Maiano, P. Bosland, M. Ellis, P. Michelato, G. Olry, and R. Ruber, The superconducting accelerator for the ESS project, in Proceedings of the International Conference on RF Superconductivity (SRF'17), Lanzhou, China, 2017 (JACoW Publishing, Geneva, Switzerland, 2017), MOYA01, pp. 24-28.

[7] Y. Chi, S. Fu, W. Pan, P. Sha, and Q. Xing, Chinese ADS project and proton accelerator development, in Proceedings of the 26th International Linear Accelerator Conference (LINAC'12), Tel Aviv, Israel, 2012 (JACoW Publishing, Geneva, Switzerland, 2013), TU1A03.

[8] The European X-Ray Free-Electron Laser, technical design report, Technical Report No. DESY 2006-097, DESY, 2007.

[9] J. Galayda, The LCLS-II: A high power upgrade to the LCLS, in Proceedings of the 9th International Particle Accelerator Conference (IPAC'18), Vancouver, BC, 2018 (JACoW Publishing, Geneva, Switzerland, 2018), MOYGB2, pp. 18-23.

[10] The International Linear Collider Technical Design Report Volume 3.II: Accelerator Baseline Design, arXiv:1306 .6328 . 
[11] The International Linear Collider: A global project, arXiv: 1903.01629.

[12] G. Ciovati, Effect of low-temperature baking on the radio-frequency properties of niobium superconducting cavities for particle accelerators, J. Appl. Phys. 96, 1591 (2004).

[13] A. Grassellino, A. Romanenko, A. Crawford, O. Melnychuk, A. Rowe, M. Wong, C. Cooper, D. Sergatskov, D. Bice, Y. Trenikhina, L. D. Cooley, C. Ginsburg, and R. D. Kephart, Fermilab experience of post-annealing losses in SRF niobium cavities due to furnace contamination and the ways to its mitigation: A pathway to processing simplification and quality factor improvement, arXiv:1305.2182.

[14] D. Reschke and L. Lilje, Recent experience with nine-cell cavity performance at DESY, in Proceedings of the International Conference on RF Superconductivity (SRF'07), Beijing, China, 2007 (JACoW Publishing, Geneva, Switzerland, 2008), TUP77.

[15] A. Grassellino, A. Romanenko, D. Bice, O. Melnychuk, A. C. Crawford, S. Chandrasekaran, Z. Sung, D. A. Sergatskov, M. Checchin, S. Posen, M. Martinello, and $\mathrm{G}$. $\mathrm{Wu}$, Accelerating fields up to $49 \mathrm{MV} / \mathrm{m}$ in TESLAshape superconducting rf niobium cavities via $75 \mathrm{C}$ vacuum bake, arXiv:1806.09824.

[16] A. Grassellino, A. Romanenko, Y. Trenikhina, M. Checchin, M. Martinello, O. S. Melnychuk, S. Chandrasekaran, D. A. Sergatskov, S. Posen, A. C. Crawford, S. Aderhold, and D. Bice, Unprecedented quality factors at accelerating gradients up to $45 \mathrm{MVm}^{-1}$ in niobium superconducting resonators via low temperature nitrogen infusion, Supercond. Sci. Technol. 30, 094004 (2017).

[17] A. Grassellino, A. Romanenko, D. Sergatskov, O. Melnychuk, Y. Trenikhina, A. Crawford, A. Rowe, M. Wong, T. Khabiboulline, and F. Barkov, Nitrogen and argon doping of niobium for superconducting radio frequency cavities: A pathway to highly efficient accelerating structures, Supercond. Sci. Technol. 26, 102001 (2013).

[18] S. Posen, G. Wu, A. Grassellino, E. Harms, O. S. Melnychuk, D. A. Sergatskov, N. Solyak, A. Romanenko, A. Palczewski, D. Gonnella, and T. Peterson, Role of magnetic flux expulsion to reach $Q_{0}>3 \times 10^{10}$ in superconducting rf cryomodules, Phys. Rev. Accel. Beams 22, 032001 (2019).

[19] R. Ferdinand and P. Bertrand, Status and challenges of the spiral 2 facility, in Proceedings of the 25th International Linear Accelerator Conference, LINAC-2010, Tsukuba, Japan (KEK, Tsukuba, Japan, 2010), MO201.

[20] F. Zhu, J. Hao, S. Quan, L. Lin, F. Wang, S. Chen, M. Chen, L. Feng, A. Cheng, and K. Liu, The effect of nitrogen infusion on a $162.5 \mathrm{MHz}$ low beta superconducting HWR cavity, Nucl Instrum. Methods Phys. Res., Sect. A 937, 21 (2019).

[21] Z. Yao, P. Kolb, and R. Laxdal, Medium field Q-slope in low beta resonators, in Proceedings of the International Conference on RF Superconductivity (SRF2015), Whistler, $B C, 2015$ (JACoW Publishing, Geneva, Switzerland, 2015), WEA1A03.

[22] D. Longuevergne and A. Miyazaki, How can geometry impact the magnetic flux trapping of superconducting accelerating cavities, arXiv:2009.07007.
[23] B. Dury, Simulations of flux trapping in cavities transitioning to a super conducting state, TRIUMF internal report, 2019.

[24] J. P. Turneaure, J. Halbritter, and H. A. Schwettman, The surface impedance of superconductors and normal conductors: The Mattis-Bardeen theory, J. Supercond. 4, 341 (1991).

[25] A. Gurevich, Theory of RF superconductivity for resonant cavities, Supercond. Sci. Technol. 30, 034004 (2017).

[26] L. Szécsi, Measurement of the dependence on frequency of the residual resistance of superconducting layers of lead, $\mathrm{Z}$. Phys. A 241, 36 (1971).

[27] M. Martinello, S. Aderhold, S. Chandrasekaran, M. Checchin, A. Grassellino, O. Melnychuk, S. Posen, A. Romanenko, and D. Sergatskov, Advancement in the understanding of the field and frequency dependent microwave surface resistance of niobium, in Proceedings of the International Conference on RF Superconductivity (SRF'17), Lanzhou, China, 2017 (JACoW Publishing, Geneva, Switzerland, 2018), TUYAA02, pp. 364-367.

[28] E. Mahner, S. Calatroni, E. Chiaveri, E. Haebel, and J. M. Tessier, A new instrument to measure the surface resistance of superconducting samples at $400 \mathrm{MHz}, \mathrm{Rev}$. Sci. Instrum. 74, 3390 (2003).

[29] T. Junginger, W. Weingarten, and C. Welsch, Extension of the measurement capabilities of the quadrupole resonator, Rev. Sci. Instrum. 83, 063902 (2012).

[30] R. Kleindienst, A. Burrill, S. Keckert, J. Knobloch, and O. Kugeler, Commissioning results of the HZB quadrupole resonator, in Proceedings of the International Conference on RF Superconductivity (SRF2015), Whistler, BC, 2015 (JACoW Publishing, Geneva, Switzerland, 2015), WEA1A04, pp. 930-936.

[31] H. Park, S. De Silva, and J. Delayen, Superconducting cavity for the measurements of frequency, temperature, RF field dependence of the surface resistance, in Proceedings of the International Conference on RF Superconductivity (SRF2015), Whistler, BC, 2015 (JACoW Publishing, Geneva, Switzerland, 2015), MOPB003, pp. 70-73.

[32] H. Park, S. D. Silva, and J. Delayen, Measurement of surface resistance properties with coaxial resonators Review, in Proceedings of the International Conference on RF Superconductivity (SRF2019), Dresden, Germany, 2019 (JACoW Publishing, Geneva, Switzerland, 2019), TUFUB7, pp. 374-380.

[33] A. Gurevich, Multiscale mechanisms of SRF breakdown, Physica (Amsterdam) 441C, 38 (2006).

[34] A. Gurevich, Thermal RF breakdown of superconducting cavities, ICFA Beam Dyn. Newslett. 39, 34 (2006), https:// inspirehep.net/literature/717103.

[35] P. Bauer, N. Solyak, G. Ciovati, G. Eremeev, A. Gurevich, L. Lilje, and B. Visentin, Evidence for non-linear BCS resistance in SRF cavities, Physica (Amsterdam) 441C, 51 (2006).

[36] W. Weingarten, Field-dependent surface resistance for superconducting niobium accelerating cavities, Phys. Rev. Accel. Beams 14, 101002 (2011).

[37] V. Palmieri, The problem of Q-drop in superconducting resonators revised by the analysis of fundamental concepts from RF-superconductivity theory, in Proceedings of the 
2005 Workshop on RF Superconductivity (SRF'05), Ithaca, New York, 2005 (JACoW Publishing, Geneva, Switzerland, 2006), TUA02.

[38] T. Kubo and A. Gurevich, Field-dependent nonlinear surface resistance and its optimization by surface nanostructuring in superconductors, Phys. Rev. B 100, 064522 (2019).

[39] R. Laxdal, R. Lee, and M. Pasini, Progress in the Conceptual Design of the ISAC-II Linac at TRIUMF, in Proceedings of the 19th Particle Accelerator Conference, Chicago, IL, 2001 (IEEE, Piscataway, NJ, 2001), FPAH111.

[40] Z. Yao, T. Junginger, R. Laxdal, B. Matheson, B. Waraich, and V. Zvyagintsev, Design of multi-frequency coaxial test resonators, in Proceedings of the International Conference on RF Superconductivity (SRF'17), Lanzhou, China, 2017 (JACoW Publishing, Geneva, Switzerland, 2018), TUPB065, pp. 531-534.

[41] Z. Yao, J. Keir, P. Kolb, A. Kong, R. Laxdal, B. Matheson, E. Thoeng, B. Waraich, Q. Zheng, and V. Zvyagintsev, Tests of multi-frequency coaxial resonators, in Proceedings of the 29th Linear Accelerator Conference (LINAC'18), Beijing, China, 2018 (JACoW Publishing, Geneva, Switzerland, 2019), TUPO040, pp. 420-422.

[42] P. Dhakal, G. Ciovati, W. Rigby, J. Wallace, and G. R. Myneni, Design and performance of a new induction furnace for heat treatment of superconducting radiofrequency niobium cavities, Rev. Sci. Instrum. 83, 065105 (2012).

[43] J.-M. Vogt, O. Kugeler, and J. Knobloch, Impact of cooldown conditions at Tc on the superconducting rf cavity quality factor, Phys. Rev. Accel. Beams 16, 102002 (2013).

[44] G. Wu, A. Grassellino, E. Harms, N. Solyak, A. Romanenko, C. Ginsburg, and R. Stanek, Achievement of ultrahigh quality factor in prototype cryomodule for LCLS-II, arXiv:1812.09368.

[45] Bartington Instruments, Whiney, Oxon, United Kingdom, http://www.bartington.com.

[46] NI National Instruments, Austin, TX, http://www.ni.com.

[47] Advantage Engineering, Inc., Greenwood, IN, http://www .advantageengineering.com.

[48] M. Laverty, K. Fong, and Q. Zheng, TRIUMF e-linac RF control system design, in Proceedings of the 23rd Particle Accelerator Conference, Vancouver, Canada, 2009 (IEEE, Piscataway, NJ, 2009), FPAH111.

[49] BEXT Inc., San Diego, CA, http://www.bext.com.

[50] R\&K Company Limited, Fuji-City, Shizuoka-Pref., 4168577 Japan, http://www.rk-microwave.com.

[51] J. Holzbauer, C. Contreras, Y. Pischalnikov, D. Sergatskov, and W. Schappert, Improved RF measurements of SRF cavity quality factors, Nucl. Instrum. Methods Phys. Res., Sect. A 913, 7 (2019).

[52] A. Romanenko, Surface characterization of $\mathrm{Nb}$ cavity sections-Understanding the high field Q-slope, Ph.D. thesis, Cornell University, 2009.

[53] COMSOL Multiphysics v.5.4. COMSOL AB, Stockholm, Sweden, http://www.comsol.com.
[54] J. Maniscalco and M. Liepe, A computational method for more accurate measurements of the surface resistance in SRF cavities, in Proceedings of the 9th International Particle Accelerator Conference (IPAC'18), Vancouver, $B C, 2018$ (JACoW Publishing, Geneva, Switzerland, 2018), WEPMF042, pp. 2458-2460.

[55] D. Longuevergne, Geometrical corrections for accurate fitting of the field dependent surface resistance for superconducting accelerating cavities, Nucl. Instrum. Methods Phys. Res., Sect. A 910, 41 (2018).

[56] R. Kleindienst, S. Keckert, J. Knobloch, and O. Kugeler, Advanced method to extract the surface resistance from $Q_{0}$ measurements, in Proceedings of the International Conference on RF Superconductivity (SRF'17), Lanzhou, China, 2017 (JACoW Publishing, Geneva, Switzerland, 2018), THPB054, pp. 867-869.

[57] J. R. Delayen, H. Park, S. U. De Silva, G. Ciovati, and Z. $\mathrm{Li}$, Determination of the magnetic field dependence of the surface resistance of superconductors from cavity tests, Phys. Rev. Accel. Beams 21, 122001 (2018).

[58] G. Ciovati, SUPERFIT: A computer code to fit surface resistance and penetration depth of a superconductor, Technical Report, https://doi.org/10.2172/955388.

[59] B. Visentin, Q-slope at high gradients: review of experiments and theories, in Proceedings of the 11th Workshop on RF Superconductivity (SRF'03), Lübeck/Travemünde, Germany, 2003 (JACoW Publishing, Geneva, Switzerland, 2004), TUO01.

[60] E. Thoeng, R. Baartman, R. Laxdal, B. Matheson, G. Morris, N. Muller, S. Saminathan, A. Chen, and T. Junginger, Beta-SRF - A new facility to characterize SRF materials near fundamental limits, in Proceedings of the 9th International Particle Accelerator Conference (IPAC'18), Vancouver, BC, 2018 (JACoW Publishing, Geneva, Switzerland, 2018), THPML122, pp. 4961-4963.

[61] A. Romanenko, A. Grassellino, F. Barkov, and J. P. Ozelis, Effect of mild baking on superconducting niobium cavities investigated by sequential nanoremoval, Phys. Rev. Accel. Beams 16, 012001 (2013).

[62] H. Padamsee, J. Knobloch, and T. Hays, RF Superconductivity for Accelerators, 2nd ed. (Wiley, New York, 2008).

[63] P. Dhakal, G. Ciovati, and A. Gurevich, Flux expulsion in niobium superconducting radio-frequency cavities of different purity and essential contributions to the flux sensitivity, Phys. Rev. Accel. Beams 23, 023102 (2020).

[64] A. Grassellino, C. Beard, P. Kolb, R. Laxdal, N. S. Lockyer, D. Longuevergne, and J. E. Sonier, Muon spin rotation studies of niobium for superconducting rf applications, Phys. Rev. Accel. Beams 16, 062002 (2013).

[65] T. Junginger, S. H. Abidi, R. D. Maffett, T. Buck, M. H. Dehn, S. Gheidi, R. Kiefl, P. Kolb, D. Storey, E. Thoeng, W. Wasserman, and R. E. Laxdal, Field of first magnetic flux entry and pinning strength of superconductors for $\mathrm{rf}$ application measured with muon spin rotation, Phys. Rev. Accel. Beams 21, 032002 (2018).

[66] Origin, version 2020, OriginLab Corporation, Northampton, MA, http://www.originlab.com. 